\title{
On the two-dimensional Boussinesq equations with temperature-dependent thermal and viscosity diffusions in general Sobolev spaces
}

\author{
Zihui He and Xian Liao(i)
}

\begin{abstract}
We study the existence, uniqueness as well as regularity issues for the two-dimensional incompressible Boussinesq equations with temperature-dependent thermal and viscosity diffusion coefficients in general Sobolev spaces. The optimal regularity exponent ranges are considered.
\end{abstract}

Mathematics Subject Classification. 35Q30, 76D03.

Keywords. Boussinesq equations, Temperature-dependent diffusion coefficients, Existence, Uniqueness, Regularity, Sobolev spaces.

\section{Introduction}

In the present paper, we consider the two-dimensional incompressible Boussinesq equations

$$
\left\{\begin{array}{l}
\partial_{t} \theta+u \cdot \nabla_{x} \theta-\operatorname{div}_{x}\left(\kappa \nabla_{x} \theta\right)=0, \\
\partial_{t} u+u \cdot \nabla_{x} u-\operatorname{div}_{x}\left(\mu S_{x} u\right)+\nabla_{x} \Pi=\beta \theta \overrightarrow{e_{2}}, \\
\operatorname{div}_{x} u=0,
\end{array}\right.
$$

where $(t, x) \in[0, \infty) \times \mathbb{R}^{2}$ denote the time and space variables, respectively. The unknown temperature function $\theta=\theta(t, x):[0, \infty) \times \mathbb{R}^{2} \rightarrow \mathbb{R}$ satisfies the parabolic-type equation $(1.1)_{1}$, and the unknown velocity vector field $u=u(t, x):[0, \infty) \times \mathbb{R}^{2} \rightarrow \mathbb{R}^{2}$ together with the unknown pressure term $\Pi=\Pi(t, x)$ : $[0, \infty) \times \mathbb{R}^{2} \rightarrow \mathbb{R}$ satisfies the incompressible Navier-Stokes type equations $(1.1)_{2}-(1.1)_{3}$, respectively. We are going to study the well-posedness and regularity problems for the Boussinesq system (1.1) together with the initial data

$$
\left.(\theta, u)\right|_{t=0}=\left(\theta_{0}, u_{0}\right)
$$

We write $x=\left(\begin{array}{l}x_{1} \\ x_{2}\end{array}\right) \in \mathbb{R}^{2}$ with $x_{1}, x_{2}$ denoting the horizontal and vertical components, respectively. Let $u=\left(\begin{array}{l}u^{1} \\ u^{2}\end{array}\right):[0, \infty) \times \mathbb{R}^{2} \rightarrow \mathbb{R}^{2}$, and let

$$
\frac{1}{2} S_{x} u:=\frac{1}{2}\left(\nabla_{x} u+\left(\nabla_{x} u\right)^{T}\right), \text { with } \nabla_{x} u=\left(\partial_{x_{j}} u^{i}\right)_{1 \leq i, j \leq 2}
$$

denote the symmetric deformation tensor in the second equation $(1.1)_{2}$ above. The vector field $\overrightarrow{e_{2}}$ denotes the unit vector in the vertical direction: $\overrightarrow{e_{2}}=\left(\begin{array}{l}0 \\ 1\end{array}\right)$, and $\beta \theta \overrightarrow{e_{2}}$ stands for the buoyancy force, with the constant parameter $\beta>0$ denoting the thermodynamic dilatation coefficient which will be assumed to be 1 in the following context for simplicity. 
We consider the cases when the heat diffusion and the viscosity in the fluids are sensitive to the change of temperatures, that is, the thermal diffusivity $\kappa$ and the viscosity coefficient $\mu$ may depend on the temperature function $\theta$ as follows

$$
\kappa=a(\theta), \quad \mu=b(\theta), \quad \text { with } \kappa_{*} \leq a \leq \kappa^{*}, \mu_{*} \leq b \leq \mu^{*},
$$

where $\kappa_{*} \leq \kappa^{*}, \mu_{*} \leq \mu^{*}$ are positive constants. We will not assume any smallness conditions on $\kappa^{*}-\kappa_{*}$ or $\mu^{*}-\mu_{*}$, and large variations in these diffusivity coefficients are permitted.

The Boussinesq system (1.1) arises from the zero order approximation to the corresponding inhomogeneous hydrodynamic systems, which are nonlinear coupling between the Navier-Stokes equations or Euler equations and the thermodynamic equations for the temperature or density functions: The Boussinesq approximation [5] ignores density differences except when they appear in the buoyancy term. They are common geophysical models describing the dynamics from large-scale atmosphere and ocean flows to solar and plasma inner convection, where density stratification is a typical feature [22,34].

The temperature or density differences in the inhomogeneous fluids may cause density gradients. When the thermodynamical coefficients such as the heat conducting coefficients and the viscosity coefficients are assumed to be constant in the Boussinesq approximation [i.e., $\kappa, \mu$ are constants in (1.1)], density gradients influence the motion of the flows only through the buoyancy force, which may lead to finite time singularity in the flows (the formation of the finite time singularity is sensitive to the thermal and viscous dissipation and see Sect. 1.1 below for more references on this topic).

However, the temperature variations do influence the thermal conductivity and the viscosity coefficients effectively, even for simple fluids such as pure water $[32$, Sect. 6$] .{ }^{1},{ }^{2}$ In many applications in the engineering, one also aims for effective thermal conductivities in building thermal energy storage materials [21]. Therefore in plenty of physical models, density gradients would influence the motion of the fluids not only through buoyancy force, but also through the variations of the diffusion coefficients. It is then interesting to study the well-posedness and regularity problems of the Boussinesq system (1.1)-(1.3).

\subsection{Known results}

The well-posedness and regularity problems on the two-dimensional Boussinesq equations have attracted considerable attention from the PDE community. Many interesting mathematical results have been established in the past two decades, mainly in the cases with constant thermal diffusivity coefficient $\kappa$ and viscosity coefficient $\mu$ :

$$
\left\{\begin{array}{l}
\partial_{t} \theta+u \cdot \nabla_{x} \theta-\kappa \Delta_{x} \theta=0, \\
\partial_{t} u+u \cdot \nabla_{x} u-\mu \Delta_{x} u+\nabla_{x} \Pi=\theta \overrightarrow{e_{2}}, \\
\operatorname{div}_{x} u=0 \\
\left.(\theta, u)\right|_{t=0}=\left(\theta_{0}, u_{0}\right) .
\end{array}\right.
$$

If $\kappa=\mu=0$, the two-dimensional inviscid Boussinesq equations (1.4) can be compared with the three-dimensional incompressible axisymmetric Euler equations with swirl, where the buoyancy force corresponds to the vortex stretching mechanism [35]. The local-in-time well-posedness as well as some

\footnotetext{
${ }^{1}$ The absolute viscosity of the water under nominal atmospheric pressure in units of millipascal seconds is given by $1.793\left(0^{\circ}\right), 0.547\left(50^{\circ}\right), 0.282\left(100^{\circ}\right)$, respectively [32, pp. 6-186]. The thermal conductivity of the water under nominal atmospheric pressure in units of Watt per meter kelvin is given by $0.5562\left(0^{\circ}\right), 0.6423\left(50^{\circ}\right), 0.6729\left(100^{\circ}\right)$, respectively [32, Page 6-214].

${ }^{2}$ It is common to adapt the exponential viscosity law $\mu(T)=C_{1} \exp \left(C_{2} /\left(C_{3}+T\right)\right)$ and quasi-constant heat conductivity law $\kappa(T)=C_{4}$ for the liquids, while the viscosity law $\mu(T)=\left(\mu\left(T_{m}\right)\right) \frac{T}{T_{m}} \frac{T_{m}+C_{5}}{T+C_{6}}$ and the thermal conductivity law $\kappa(T)=C_{6} \mu(T)$ for the gases, where $T$ denotes the absolute temperature, $T_{m}$ denotes the reference temperature, and $C_{j}$, $1 \leq j \leq 6$ are positive constants $[37, \mathrm{I}]$.
} 
blowup criteria have been well known for decades, see, e.g., $[10,11,42]$. We mention that an (improved) lower bound for the lifespan which tends to infinity as the initial temperature tends to a constant (and correspondingly, as the initial swirl tends to zero for the 3D axisymmetric Euler equations) was given in [11]. The fundamental global regularity problem for the 2D inviscid Boussinesq equations remains still open. Recently, an interesting example of finite-energy strong solutions with a finite weighted Hölder norm in a wedge-shaped domain, which become singular at the origin in finite time, has been given in [19] (see also an interesting example of solutions in Hölder-type spaces with finite-time singularity for 3D axisymmetric Euler equations in [18]).

If $\kappa>0$ and $\mu>0$ are positive constants, on the contrary, the convection terms can be controlled thanks to the strong diffusion effects, and the global-in-time existence and regularity results can be established (see, e.g., [7]). Particular interests then raised if only partial dissipation is present, that is, either $\kappa=0$ whereas $\mu>0$ or $\kappa>0$ whereas $\mu=0$ (see, e.g., H.K. Moffatt's list of the twenty first century PDE problems [36]). The global-in-time results continue to hold, thanks to a priori estimates in the $L^{p}$-framework as well as the sharp Sobolev embedding inequality in dimension two with a logarithm correction, which help the partial diffusion terms to control the demanding term $\partial_{x_{1}} \theta$ successfully (see $[9,27]$ and see $[24]$ for less regular cases). Further developments were made for horizontal dissipation cases (see, e.g., [13]), for vertical dissipation cases (see, e.g., [8]), and for the fractional dissipation cases (see, e.g., $[25,26])$. See the review notes [43] and the references therein for more interesting results and sketchy proofs.

There also have been remarkable progresses in solving the two dimensional Boussinesq equations (1.1)-(1.3) when the thermal and viscosity diffusion coefficients $\kappa, \mu$ are variable and depend smoothly on the unknown temperature function $\theta$. In the variational formulation framework, the global-in-time existence of a solution of (1.1)-(1.3) has been established in [17] [see [20] for a similar formulation of (1.1)-(1.3)] for the motion of the so-called Bingham fluid (as a non-Newtonian fluid), where $\kappa$ is a positive constant, $\beta=0$ and $\mu$ depends not only on $\theta$ but also on $S u /|S u|$. The Boussinesq-Stefan model has been investigated in [38], where the phase transition was taken into account. The global-in-time existence and the uniqueness of the solutions for (1.1)-(1.3) have been shown in $[15,23,33]$ under Dirichlet boundary conditions and in [37] under generalized outflow boundary conditions. We remark that the resolution of the nonhomogeneous Boussinesq system under more physical boundary conditions (e.g., with Dirichlet boundary conditions only on the inflow part of the boundary while with no prescribed assumptions on the outflow part) remains unsolved.

Lorca and Boldrini [33] (see also [15,23]) studied the initial-boundary value problem of the Boussinesq system (1.1)-(1.3) in dimension two and three under the initial condition (1.2) and Dirichlet boundary conditions, and obtained a global-in-time weak solution

$$
(\theta, u) \in\left(L_{\mathrm{loc}}^{\infty}\left([0, \infty) ; L^{2}(\Omega)\right)\right)^{3}
$$

as well as a local-in-time unique strong solution

$$
(\theta, u) \in L_{\mathrm{loc}}^{\infty}\left([0, \infty) ; H^{2}(\Omega)\right) \times\left(L_{\mathrm{loc}}^{\infty}\left([0, \infty) ; H^{1}(\Omega)\right)^{2} .\right.
$$

The remarkable global-in-time existence and uniqueness results of the smooth solutions

$$
(\theta, u) \in\left(L_{\text {loc }}^{\infty}\left([0, \infty) ; H^{s}\left(\mathbb{R}^{2}\right)\right) \cap L_{\text {loc }}^{2}\left([0, \infty) ; H^{s+1}\left(\mathbb{R}^{2}\right)\right)\right)^{3}, \quad s>2
$$

have been successfully established by Wang and Zhang [41], which affirms the propagation of high regularities (without finite time singularity) of the two dimensional Boussinesq flow in the presence of viscosity variations (see [39] for the case $s=2$ ). We remark that the $L_{x}^{2}$-norm of the velocity vector field may grow in time due to the buoyancy forcing term, even provided with constant diffusion coefficients and smooth and fast decaying small initial data [6], and hence the norm with respect to the time variable in (1.5) and (1.6) is only locally in time.

It is still not clear whether there will be finite time singularity for the two-dimensional Boussinesq flow (1.1)-(1.3) in the presence of viscosity variations while no heat diffusion (i.e., $\kappa=0, \mu=b(\theta)$ ), 
and we mention a recent work [2] toward this direction in the case of less heat diffusion (with $\operatorname{div}(\kappa \nabla \theta)$ replaced by $(-\Delta)^{1 / 2}$ ) and the small viscosity variation assumption: $|\mu-1| \leq \varepsilon$. A closely related question would pertain to the global-in-time well-posedness problem of the two-dimensional inhomogeneous incompressible Navier-Stokes equations with density-dependent viscosity coefficient

$$
\left\{\begin{array}{l}
\partial_{t} \rho+u \cdot \nabla_{x} \rho=0, \\
\partial_{t}(\rho u)+\operatorname{div}_{x}(\rho u \otimes u)-\operatorname{div}_{x}\left(\mu S_{x} u\right)+\nabla_{x} \Pi=0, \\
\operatorname{div}_{x} u=0, \\
\left.(\rho, \rho u)\right|_{t=0}=\left(\rho_{0}, m_{0}\right) .
\end{array}\right.
$$

The global-in-time existence results of weak solutions of (1.7) (see, e.g., [3,31]) as well as the local-in-time well-posedness results (see, e.g., [28]) have been well known, while the global-in-time regularities still remain open (see, e.g., $[1,16]$ for some interesting results under the assumption on the weak inhomogeneity).

To the best of our knowledge, there are no global-in-time regularity propagation results by the twodimensional Boussinesq flow with temperature-dependent diffusion coefficients (1.1)-(1.2)-(1.3) in the low regularity regime

$$
H^{s}, \quad s<2,
$$

or in the general Sobolev setting

$$
\theta_{0} \in H_{x}^{s_{\theta}}\left(\mathbb{R}^{2}\right), \quad u_{0} \in\left(H_{x}^{s_{u}}\left(\mathbb{R}^{2}\right)\right)^{2}
$$

with different regularity indices $s_{\theta}$ and $s_{u}$. In this paper, we are going to investigate the existence, uniqueness as well as the regularity problems in these general Sobolev functional settings.

To conclude this subsection, let us just mention some recent interesting progresses on the stability of the stationary shear flow solutions (together with the corresponding striated temperature function) to the Boussinesq equations (1.4), with full dissipation or partial dissipation, in, e.g., [14,40,44] and references therein. It should also be interesting to investigate the stability of the stationary striated solutions of the Boussinesq equations with variable diffusion coefficients (1.1). We mention a recent work in this direction on the incompressible Navier-Stokes equations with constant density function but with variable viscosity coefficient [30].

\subsection{Main results}

We are going to show the global-in-time existence of weak solutions to the Cauchy problem for the Boussinesq system (1.1)-(1.2)-(1.3) in the whole two-dimensional space $\mathbb{R}^{2}$ under the low-regularity initial condition $\left(\theta_{0}, u_{0}\right) \in L^{2}\left(\mathbb{R}^{2}\right) \times\left(L^{2}\left(\mathbb{R}^{2}\right)\right)^{2}$. The uniqueness result holds true if the initial temperature function becomes smoother $\left(\theta_{0}, u_{0}\right) \in H^{1}\left(\mathbb{R}^{2}\right) \times\left(L^{2}\left(\mathbb{R}^{2}\right)\right)^{2}$. Finally, we will establish the global-in-time regularity of the solutions in the general Sobolev setting $\left(\theta_{0}, u_{0}\right) \in H^{s_{\theta}}\left(\mathbb{R}^{2}\right) \times\left(H^{s_{u}}\left(\mathbb{R}^{2}\right)\right)^{2} \subset H^{1}\left(\mathbb{R}^{2}\right) \times$ $\left(L^{2}\left(\mathbb{R}^{2}\right)\right)^{2}$ with the restriction $s_{u}-1 \leq s_{\theta} \leq s_{u}+2$. These regularity exponent ranges are optimal for the existence, uniqueness and regularity results, respectively, by view of the formulations of the Boussinesq equations (1.1) with temperature-dependent diffusion coefficients (see Remark 1.3 below for more details).

We first define the weak solutions as follows.

Definition 1.1. (Weak solutions) We say that a pair $(\theta, u)$ is a weak solution of the Boussinesq equations (1.1)-(1.3) with the given initial data $\left(\theta_{0}, u_{0}\right) \in\left(L^{2}\left(\mathbb{R}^{2}\right)\right)^{3}$ if the following statements hold:

- The temperature function

$$
\theta=\theta(t, x) \in C\left([0, \infty) ; L_{x}^{2}\left(\mathbb{R}^{2}\right)\right) \cap L_{\mathrm{loc}}^{2}\left([0, \infty) ; H_{x}^{1}\left(\mathbb{R}^{2}\right)\right)
$$


satisfies the initial condition $\left.\theta\right|_{t=0}=\theta_{0}$, the energy equality

$$
\frac{1}{2}\|\theta(T, \cdot)\|_{L_{x}^{2}\left(\mathbb{R}^{2}\right)}^{2}+\int_{0}^{T} \int_{\mathbb{R}^{2}}\left(\kappa|\nabla \theta|^{2}\right)(t, x) \mathrm{d} x \mathrm{dt}=\frac{1}{2}\|\theta(0, \cdot)\|_{L_{x}^{2}\left(\mathbb{R}^{2}\right)}^{2},
$$

for all positive times $T>0$, and the equation

$$
\partial_{t} \theta+u \cdot \nabla \theta-\operatorname{div}_{x}(\kappa \nabla \theta)=0
$$

in $L_{\text {loc }}^{2}\left([0, \infty) ; H_{x}^{-1}\left(\mathbb{R}^{2}\right)\right)$.

- The velocity vector field

$$
u=u(t, x) \in C\left([0, \infty) ;\left(L_{x}^{2}\left(\mathbb{R}^{2}\right)\right)^{2}\right) \cap L_{\mathrm{loc}}^{2}\left([0, \infty) ;\left(H_{x}^{1}\left(\mathbb{R}^{2}\right)\right)^{2}\right)
$$

satisfies the initial condition $\left.u\right|_{t=0}=u_{0}$, the divergence-free condition $\operatorname{div}_{x} u=0$, the energy equality

$$
\begin{aligned}
& \frac{1}{2}\|u(T, \cdot)\|_{L_{x}^{2}\left(\mathbb{R}^{2}\right)}^{2}+\frac{1}{2} \int_{0}^{T} \int_{\mathbb{R}^{2}}\left(\mu|S u|^{2}\right)(t, x) \mathrm{d} x \mathrm{dt} \\
& =\frac{1}{2}\|u(0, \cdot)\|_{L_{x}^{2}\left(\mathbb{R}^{2}\right)}^{2}+\int_{0}^{T} \int_{\mathbb{R}^{2}}\left(\theta u_{2}\right)(t, x) \mathrm{d} x \mathrm{dt}, \quad \forall T>0,
\end{aligned}
$$

and the equation

$$
\partial_{t} u+u \cdot \nabla_{x} u-\operatorname{div}_{x}\left(\mu S_{x} u\right)+\nabla_{x} \Pi=\theta \overrightarrow{e_{2}}
$$

in $L_{\text {loc }}^{2}\left([0, \infty) ;\left(H_{x}^{-1}\left(\mathbb{R}^{2}\right)\right)^{2}\right)$ for some scalar function $\Pi \in L_{\text {loc }}^{2}\left([0, \infty) \times \mathbb{R}^{2}\right)$ with $\nabla \Pi \in L_{\text {loc }}^{2}([0, \infty)$; $\left(H_{x}^{-1}\left(\mathbb{R}^{2}\right)\right)^{2}$ ) and $\int_{B_{1}} \Pi \mathrm{d} x=0$ a.e. $t$ (with $B_{1}$ denoting the unit disk in $\mathbb{R}^{2}$ ).

For any fixed $T>0, p \geq 1, q \geq 1, s \geq 0$ and for any fixed (vector-valued) function $f:[0, T] \times \mathbb{R}^{2} \mapsto \mathbb{R}^{m}$, $m \geq 1$, we denote

$$
\|f\|_{L_{T}^{p} X_{x}}:=\|\| f(t)\left\|_{X_{x}\left(\mathbb{R}^{2} ; \mathbb{R}^{m}\right)}\right\|_{L_{t}^{p}([0, T])} \text { with } X=H^{s} \text { or } L^{q} .
$$

The functional space $L^{p}\left([0, T] ; H^{s}\left(\mathbb{R}^{2} ; \mathbb{R}^{m}\right)\right)$ consists of all functions $f:[0, \infty) \times \mathbb{R}^{2} \rightarrow \mathbb{R}^{m}$ satisfying $\|f\|_{L_{T}^{p} H_{x}^{s}}<\infty$. We have the following existence, uniqueness as well as global-in-time regularity results for the solutions of the Cauchy problem for the Boussinesq equations (1.1)-(1.2)-(1.3) on the whole twodimensional space $\mathbb{R}^{2}$.

Theorem 1.2. (Existence, uniqueness and global-in-time regularity) For any initial data $\theta_{0} \in L^{2}\left(\mathbb{R}^{2}\right)$ and $u_{0} \in\left(L^{2}\left(\mathbb{R}^{2}\right)\right)^{2}$, there exists a global-in-time weak solution

$$
(\theta, u) \in C\left([0, \infty) ;\left(L^{2}\left(\mathbb{R}^{2}\right)\right)^{3}\right) \cap L_{l o c}^{2}\left([0, \infty) ;\left(H^{1}\left(\mathbb{R}^{2}\right)\right)^{3}\right)
$$

of the initial value problem (1.1)-(1.2)-(1.3).

If $\theta_{0} \in H^{1}\left(\mathbb{R}^{2}\right), u_{0} \in\left(L^{2}\left(\mathbb{R}^{2}\right)\right)^{2}$ and the functions $a \in C_{b}^{2}\left(\mathbb{R} ;\left[\kappa_{*}, \kappa^{*}\right]\right), b \in C_{b}^{2}\left(\mathbb{R} ;\left[\mu_{*}, \mu^{*}\right]\right)$ have finite first and second derivatives, then the weak solution is indeed unique, and satisfies

$$
\theta \in C\left([0, \infty) ; H^{1}\left(\mathbb{R}^{2}\right)\right) \cap L_{\text {loc }}^{2}\left([0, \infty) ; H^{2}\left(\mathbb{R}^{2}\right)\right),
$$

as well as the following energy estimates for any given $T>0$,

$$
\begin{aligned}
& \|u\|_{L_{T}^{\infty} L_{x}^{2}}^{2}+\|\nabla u\|_{L_{T}^{2} L_{x}^{2}}^{2} \leq C\left(T\left\|\theta_{0}\right\|_{L^{2}}^{2}+\left\|u_{0}\right\|_{L^{2}}^{2}\right), \\
& \|\theta\|_{L_{T}^{\infty} H_{x}^{1}}^{2}+\left\|\left(\partial_{t} \theta, \nabla^{2} \theta\right)\right\|_{L_{T}^{2} L_{x}^{2}}^{2} \\
& \leq C\left\|\theta_{0}\right\|_{H^{1}}^{2}\left(1+\left\|\nabla \theta_{0}\right\|_{L^{2}}^{2}\right) \exp \left(C\left(T^{2}\left\|\theta_{0}\right\|_{L^{2}}^{4}+\left\|u_{0}\right\|_{L^{2}}^{4}\right)\right),
\end{aligned}
$$

where $C$ is a positive constant depending only on $\|a\|_{\mathrm{Lip}}, \kappa_{*}, \kappa^{*}, \mu_{*}$. 


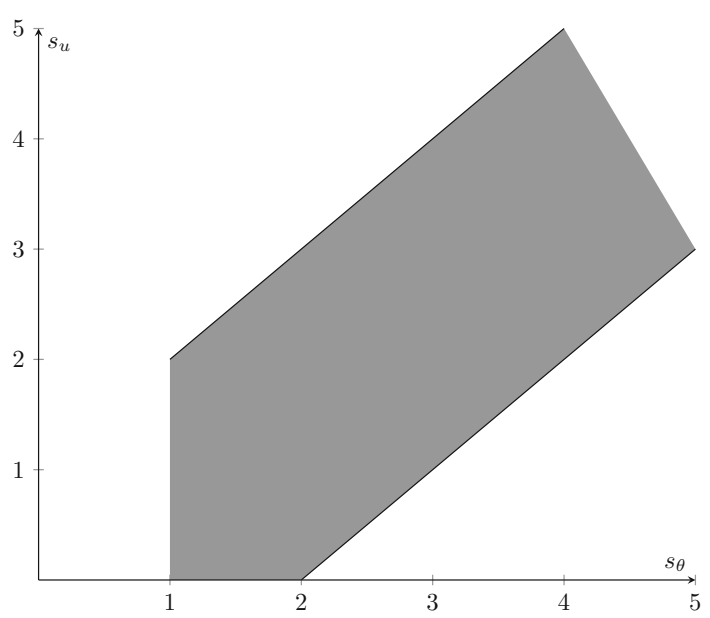

FIG. 1. Admissible regularity exponents

Furthermore, the general $H^{s}$-regularities can be propagated globally in time in the following sense: For any initial data (see the gray closed unbounded quadrangle in Fig. 1 for the admissible regularity exponent range)

$$
\begin{aligned}
& \left(\theta_{0}, u_{0}\right) \in H^{s_{\theta}}\left(\mathbb{R}^{2}\right) \times\left(H^{s_{u}}\left(\mathbb{R}^{2}\right)\right)^{2} \\
& \text { with }\left(s_{\theta}, s_{u}\right) \in\left\{\left(s_{\theta}, s_{u}\right) \subset[1, \infty) \times[0, \infty) \mid s_{u}-1 \leq s_{\theta} \leq s_{u}+2\right\}
\end{aligned}
$$

and the functions $a \in C_{b}^{2} \cap C^{\left[s_{\theta}\right]+1}, b \in C_{b}^{2} \cap C^{\left[s_{u}\right]+1}$, the unique solution $(\theta, u)$ stays in

$$
C\left([0, \infty) ; H^{s_{\theta}}\left(\mathbb{R}^{2}\right) \times\left(H^{s_{u}}\left(\mathbb{R}^{2}\right)\right)^{2}\right) \cap L_{l o c}^{2}\left([0, \infty) ; H^{s_{\theta}+1}\left(\mathbb{R}^{2}\right) \times\left(H^{s_{u}+1}\left(\mathbb{R}^{2}\right)\right)^{2}\right) .
$$

Theorem 1.2 will be proved in Sect. 2. The proof of the existence of weak solutions is rather standard, and we are going to sketch the proof in Sect. 2.1 for the reason of completeness, as we did not find the proof in the literature. As mentioned before, some well-posedness results have already been established for smooth data in the bounded domain case (see (1.5) above in, e.g., $[15,17,23,33]$ ) or in smoother functional frameworks in the whole space case (see (1.6) above in, e.g., [41]). We are going to focus on the proofs of the uniqueness result and the global-in-time regularity result (in the low regularity regimes) in Sects. 2.2 and 2.3, respectively, where different regularity exponents for different unknowns are permitted. The commutator estimates as well as the composition estimates in Lemma 2.1 will play an important role, and the a priori estimates for a general linear parabolic equation in Lemma 2.2 will be of independent interest.

We conclude this introduction part with several remarks on the results in Theorem 1.2.

Remark 1.3. (Optimality of the regularity exponent ranges in Theorem 1.2) We are going to follow the standard procedure to show the existence of weak solutions for $L^{2}$-initial data by use of the a priori energy (in)equalities (1.8) and (1.10) (see Sect. 2.1 below).

If we take the difference between two different weak solutions $\left(\theta_{1}, u_{1}\right)$ and $\left(\theta_{2}, u_{2}\right)$, the difference of the nonlinear viscosity term $\operatorname{div}(\mu S u)$ in the $u$-equation will become

$$
\operatorname{div}\left(\left(\mu_{1}-\mu_{2}\right) S u_{1}\right)+\operatorname{div}\left(\mu_{2} S\left(u_{1}-u_{2}\right)\right),
$$

which stays in $L_{\text {loc }}^{2}\left([0, \infty) ;\left(H^{-1}\left(\mathbb{R}^{2}\right)\right)^{2}\right)$ provided with

$$
\begin{aligned}
& u_{1} \text { and }\left(u_{1}-u_{2}\right) \in L_{\mathrm{loc}}^{2}\left([0, \infty) ;\left(H^{1}\left(\mathbb{R}^{2}\right)\right)^{2}\right), \\
& \text { and } \mu_{1}-\mu_{2} \in L_{\mathrm{loc}}^{\infty}\left([0, \infty) ; H^{1}\left(\mathbb{R}^{2}\right)\right) \subset L_{\mathrm{loc}}^{\infty}\left([0, \infty) ;\left(L^{1}\left(\mathbb{R}^{2}\right)\right)^{\prime}=\operatorname{BMO}\left(\mathbb{R}^{2}\right)\right) .
\end{aligned}
$$


Therefore in order to ensure the $L_{x}^{2}$-Estimate for the velocity difference $\left(u_{1}-u_{2}\right)$, we require the $H_{x}^{1}$ Estimate for the temperature difference in $\left(\mu_{1}-\mu_{2}\right)$. And hence the initial condition $\theta_{0} \in H_{x}^{1}$ (i.e., $s_{\theta} \geq 1$ above) is required for the proof of the uniqueness result (see Sect. 2.2 below).

Under the lower-regularity assumption $\theta_{0} \in H_{x}^{s}$ with $0<s<1$, the coefficients $\kappa, \mu$ are not expected to be continuous uniformly in time, and hence no uniqueness or $H^{s}$-regularity results for $\theta$ or $H^{s_{1}}, s_{1}>0$ regularity results for $u$ are expected. Nevertheless with constant diffusion coefficients (e.g., $\kappa=\mu=1$ ), the uniqueness result for the weak solutions holds true by virtue of the $L_{x}^{2}$-energy (in)equalities (similar as the classical global-in-time well-posedness result for the classical two dimensional incompressible NavierStokes equations). Furthermore, if $\kappa=1$ is a positive constant, then the $H_{x}^{s}, s \in(0,1)$-Estimate for $\theta$ holds true, provided with $u \in L_{\text {loc }}^{4}\left(L_{x}^{4}\left(\mathbb{R}^{2}\right)\right)^{2}$ (or with $\left.u_{0} \in\left(L^{2}\left(\mathbb{R}^{2}\right)\right)^{2}\right)$, simply by an interpolation argument between (1.8) and (1.14). Similarly if $\mu=1$ is a positive constant, then the $H_{x}^{s}, s>0$ Estimate for $u$ holds true, provided with $\theta \in L_{\mathrm{loc}}^{2}\left(H_{x}^{s-1}\left(\mathbb{R}^{2}\right)\right)$. Thus with constant diffusion coefficients (e.g., $\kappa=\mu=1$ ), the Sobolev regularities

$$
\left(\theta_{0}, u_{0}\right) \in\left(H^{s}\left(\mathbb{R}^{2}\right)\right) \times\left(L^{2}\left(\mathbb{R}^{2}\right)\right)^{2} \text { or }\left(L^{2}\left(\mathbb{R}^{2}\right)\right) \times\left(H^{s}\left(\mathbb{R}^{2}\right)\right)^{2}, \quad 0<s \leq 1
$$

can be propagated globally in time, and the admissible regularity exponent set (1.15) extends itself indeed to the closed set consisting of all nonnegative admissible regularity exponents:

$$
\left(s_{\theta}, s_{u}\right) \in\left\{\left(s_{\theta}, s_{u}\right) \subset[0, \infty) \times[0, \infty) \mid s_{u}-1 \leq s_{\theta} \leq s_{u}+2\right\} .
$$

In order to propagate the $H^{s_{\theta}}, s_{\theta} \geq 2$-regularity of $\theta$, we require the transport term $u \cdot \nabla \theta$ in the $\theta$ equation to be at least in $L_{\mathrm{loc}}^{2}\left([0, \infty) ; H_{x}^{s_{\theta}-1}\right)$, which requires $u \in L_{\mathrm{loc}}^{2}\left([0, \infty) ; H_{x}^{s_{\theta}-1}\right)$ and hence the initial assumption $u_{0} \in H^{s_{u}}$ with the restriction $s_{u} \geq s_{\theta}-2$ (as there is a gain of regularity of oder 1 when taking $L^{2}$-norm in the time variable in general). Similarly, in order the propagate the $H^{s_{u}}, s_{u} \geq 2$-regularity of $u$, we require the viscosity term $\operatorname{div}(\mu S u)$ in the $u$-equation to be at least in $L_{\mathrm{loc}}^{2}\left([0, \infty) ; H_{x}^{s_{u}-1}\right)$, which requires $\mu S u \in L_{\mathrm{loc}}^{2}\left([0, \infty) ; H_{x}^{s_{u}}\right)$ and hence the initial assumption $\theta_{0} \in H^{s_{\theta}}$ with the restriction $s_{\theta} \geq s_{u}-1$.

Remark 1.4. (Precise $H_{x}^{s}$-Estimates in the high regularity regime) The global-in-time regularity in the high regularity regime (1.15)-(1.16) follows immediately from the following borderline a priori estimates:

- If $\theta_{0} \in H^{s}\left(\mathbb{R}^{2}\right), u_{0} \in\left(L^{2}\left(\mathbb{R}^{2}\right)\right)^{2}$ with $s \in(1,2]$ and the function $a \in C_{b}^{2}(\mathbb{R})$, then for $s \in(1,2)$ it holds

$$
\begin{aligned}
& \|\theta\|_{L_{T}^{\infty} H_{x}^{s}}^{2}+\|\nabla \theta\|_{L_{T}^{2} H_{x}^{s}}^{2} \leq C\left(\kappa_{*}\right)\left\|\theta_{0}\right\|_{H_{x}^{s}}^{2} \times \\
& \quad \times \exp \left(C\left(\kappa_{*}, s,\|a\|_{C^{2}},\|\theta\|_{L_{T}^{\infty} H_{x}^{1}}\right)\left(\|\nabla u\|_{L_{T}^{2} L_{x}^{2}}^{2}+\|\nabla \theta\|_{L_{T}^{2} H_{x}^{1}}^{2}\right)\right),
\end{aligned}
$$

and for $s=2$ it holds

$$
\begin{gathered}
\|\theta\|_{L_{T}^{\infty} H_{x}^{2}}^{2}+\|\nabla \theta\|_{L_{T}^{2} H_{x}^{2}}^{2} \leq C\left(\kappa_{*},\|a\|_{C^{2}}, \kappa^{*}\right)\left\|\theta_{0}\right\|_{H^{2}}^{2}\left(1+\left\|\nabla \theta_{0}\right\|_{L^{2}}^{2}\right)^{2} \\
\quad \times \exp \left(C\left(\kappa_{*},\|a\|_{\text {Lip }}\right)\left(\|\nabla u\|_{L_{T}^{2} L_{x}^{2}}^{2}+\|u\|_{L_{T}^{4} L_{x}^{4}}^{4}+\|\nabla \theta\|_{L_{T}^{4} L_{x}^{4}}^{4}\right)\right) .
\end{gathered}
$$

- If $\theta_{0} \in H^{1}\left(\mathbb{R}^{2}\right), u_{0} \in\left(H^{s}\left(\mathbb{R}^{2}\right)\right)^{2}$ with $s \in(0,2]$ and the function $b \in C_{b}^{2}(\mathbb{R})$, then for $s \in(0,2)$ it holds

$$
\begin{gathered}
\|u\|_{L_{T}^{\infty} H_{x}^{s}}^{2}+\|\nabla u\|_{L_{T}^{2} H_{x}^{s}}^{2} \leq C\left(\mu_{*}\right)\left(\left\|u_{0}\right\|_{H_{x}^{s}}^{2}+T\left\|\theta_{0}\right\|_{L_{x}^{2}}^{2}+\|\theta\|_{L_{T}^{2} H_{x}^{s-1}}^{2}\right) \\
\quad \times \exp \left(C\left(\mu_{*}, s,\|b\|_{C^{2}},\|\theta\|_{L_{T}^{\infty} H_{x}^{1}}\right)\left(\|\nabla u\|_{L_{T}^{2} L_{x}^{2}}^{2}+\|\nabla \theta\|_{L_{T}^{2} H_{x}^{1}}^{2}\right)\right),
\end{gathered}
$$

and for $s=2$ it holds

$$
\begin{aligned}
& \|u\|_{L_{T}^{\infty} H_{x}^{2}}^{2}+\|\nabla u\|_{L_{T}^{2} H_{x}^{2}}^{2} \leq\left(\|u\|_{L_{T}^{\infty} H_{x}^{1}}^{2}+\|\nabla u\|_{L_{T}^{2} H_{x}^{1}}^{2}\right)+C\left(\mu_{*},\|b\|_{C^{2}}\right) \times \\
& \quad\left(1+\left\|\Delta u_{0}\right\|_{L_{x}^{2}}^{2}\right)\left(1+\|u\|_{L_{T}^{\infty} H_{x}^{1}}^{2}+\|\nabla u\|_{L_{T}^{2} H_{x}^{1}}^{2}\right)^{2}\left(1+\|\theta\|_{L_{T}^{\infty} H_{x}^{1}}^{2}+\|\nabla \theta\|_{L_{T}^{2} H_{x}^{1}}^{2}\right) \\
& \quad \times \exp \left(C\left(\mu_{*},\|b\|_{C^{2}}\right)\left(\|(u, \nabla \theta)\|_{L_{T}^{4} L_{x}^{4}}^{4}+\left\|\nabla^{2} \theta\right\|_{L_{T}^{2} L_{x}^{2}}^{2}\right)\right) .
\end{aligned}
$$


- If $\theta_{0} \in H^{s}\left(\mathbb{R}^{2}\right), u_{0} \in\left(H^{s-2}\left(\mathbb{R}^{2}\right)\right)^{2}$ with $s>2$ and the function $a \in C^{[s]+1}$, then for $s \in(2,3)$ it holds

$$
\begin{aligned}
& \|\theta\|_{L_{T}^{\infty} H_{x}^{s}}^{2}+\|\nabla \theta\|_{L_{T}^{2} H_{x}^{s}}^{2} \leq C\left(\kappa_{*}\right)\left\|\theta_{0}\right\|_{H_{x}^{s}}^{2} \times \\
& \quad \times \exp \left(C\left(\kappa_{*}, s, a,\|\theta\|_{L_{T}^{\infty} L_{x}^{\infty}}\right)\left(\|u\|_{L_{T}^{2} H_{x}^{s-1}}^{2}+\|\nabla \theta\|_{L_{T}^{2} L_{x}^{\infty}}^{2}\right)\right),
\end{aligned}
$$

and for $s \geq 3$ it holds

$$
\begin{aligned}
& \|\theta\|_{L_{T}^{\infty} H_{x}^{s}}^{2}+\|\nabla \theta\|_{L_{T}^{2} H_{x}^{s}}^{2} \leq C\left(\kappa_{*}, s\right)\left(\left\|\theta_{0}\right\|_{H_{x}^{s}}^{2}+\|\nabla \theta\|_{L_{T}^{\infty} L_{x}^{\infty}}^{2}\|\nabla u\|_{L_{T}^{2} H_{x}^{s-2}}^{2}\right) \\
& \quad \times \exp \left(C\left(\kappa_{*}, s, a,\|\theta\|_{L_{T}^{\infty} L_{x}^{\infty}}\right)\left(\|\nabla u\|_{L_{T}^{2} L_{x}^{\infty}}^{2}+\|\nabla \theta\|_{L_{T}^{2} L_{x}^{\infty}}^{2}\right)\right) .
\end{aligned}
$$

- If $\theta_{0} \in H^{s-1}\left(\mathbb{R}^{2}\right), u_{0} \in\left(H^{s}\left(\mathbb{R}^{2}\right)\right)^{2}$ with $s>2$ and the function $b \in C^{[s]+1}$, then for $s \in(2,3)$ it holds

$$
\begin{aligned}
& \|u\|_{L_{T}^{\infty} H_{x}^{s}}^{2}+\|\nabla u\|_{L_{T}^{2} H_{x}^{s}}^{2} \leq C\left(\mu_{*}\right)\left(\left\|u_{0}\right\|_{H_{x}^{s}}^{2}+T\|\theta\|_{L_{T}^{\infty} H_{x}^{s-1}}^{2}\right) \\
& \quad \times \exp \left(C\left(\mu_{*}, s, b,\|\theta\|_{L_{T}^{\infty} L_{x}^{\infty}}\right)\left(\|\nabla u\|_{L_{T}^{2} H_{x}^{1}}^{2}+\|\nabla \theta\|_{L_{T}^{2} H_{x}^{s-1}}^{2}\right)\right),
\end{aligned}
$$

and for $s \geq 3$ it holds

$$
\begin{aligned}
\|u\|_{L_{T}^{\infty} H_{x}^{s}}^{2}+\|\nabla u\|_{L_{T}^{2} H_{x}^{s}}^{2} & \\
\leq & C\left(\mu_{*}\right)\left(\left\|u_{0}\right\|_{H_{x}^{s}}^{2}+T\|\theta\|_{L_{T}^{\infty} H_{x}^{s-1}}^{2}+\|\nabla u\|_{L_{T}^{\infty} L_{x}^{\infty}}^{2}\|\nabla \theta\|_{L_{T}^{2} H_{x}^{s-1}}^{2}\right) \times \\
& \times \exp \left(C\left(\mu_{*}, s, b,\|\theta\|_{L_{T}^{\infty} L_{x}^{\infty}}\right)\left(\|\nabla u\|_{L_{T}^{2} L_{x}^{\infty}}^{2}+\|\nabla \theta\|_{L_{T}^{2} L_{x}^{\infty}}^{2}\right)\right) .
\end{aligned}
$$

We are going to prove the above borderline estimates one by one in Sect. 2.3 below.

Remark 1.5. ( $L^{2}$-in time Estimate V.S. $L^{1}$-in time Estimate) Instead of the classical $L_{t}^{\infty} H_{x}^{s} \cap L_{t}^{1} H_{x}^{s+2}$ type estimate in the literature, we derive $L_{t}^{\infty} H_{x}^{s} \cap L_{t}^{2} H_{x}^{s+1}$-type estimate here, since, e.g., only the $L_{t}^{2} \dot{H}_{x}^{1}$-a priori estimate for the velocity vector field is available from the energy estimates (roughly speaking, the $L_{t}^{2}$-in time norm asks less spatial regularity on the coefficients). See Lemma 2.2 below for the a priori $H_{x}^{s}, s \in(0,2)$-Estimates for a general linear parabolic equation with divergence-free $L_{t}^{2} H_{x}^{1}$-velocity vector field, which is of independent interest.

It is in general not true that $\theta \in L_{t}^{1} H_{x}^{s+2}$ (or $u \in L_{t}^{1} H_{x}^{s+2}$ ) in the low regularity regime, although it holds straightforward in the high regularity regime.

Remark 1.6. (Remarks on the smoothness assumptions on the functions $a, b$ ) It is common to assume smooth heat conductivity law and viscosity law [37, I] in fluid models.

The Lipschitz continuity assumption $a, b \in \operatorname{Lip}$ is enough for the $H^{1} \times L^{2}$-Estimates (1.13)-(1.14) in Theorem 1.2. As for the uniqueness result, due to the following $\dot{H}_{x}^{1}$-Estimate for the difference of the diffusion coefficinets

$$
\begin{aligned}
\left\|\nabla\left(a\left(\theta_{1}\right)-a\left(\theta_{2}\right)\right)\right\|_{L_{x}^{2}} \leq & \left\|\left(a^{\prime}\left(\theta_{1}\right)-a^{\prime}\left(\theta_{2}\right)\right) \nabla \theta_{1}\right\|_{L_{x}^{2}}+\left\|a\left(\theta_{2}\right) \nabla\left(\theta_{1}-\theta_{2}\right)\right\|_{L_{x}^{2}} \\
& \leq\left\|a^{\prime}\right\|_{\text {Lip }}\left\|\nabla \theta_{1}\right\|_{L_{x}^{4}}\left\|\theta_{1}-\theta_{2}\right\|_{L_{x}^{4}}+\|a\|_{L^{\infty}}\left\|\nabla\left(\theta_{1}-\theta_{2}\right)\right\|_{L_{x}^{2}},
\end{aligned}
$$

the Lipschitz continuity assumptions $a^{\prime}, b^{\prime} \in$ Lip are required.

The dependence on the function $a$ of the constants $C$ in (1.21)-(1.22) reads precisely as [similarly for the constants in (1.23)-(1.24)]

$$
\sup _{k=0, \cdots,[s]+1} \sup _{|y| \leq c\|\theta\|_{L_{T}^{\infty} L_{x}^{\infty}}}\left|\frac{d}{d y^{k}} a(y)\right|,
$$

and hence only $a \in C^{[s]+1}$ (instead of $a \in C_{b}^{[s]+1}$ ) is required.

For the integer regularity exponents, we can simply derive the energy estimates by integration by parts (instead of the application of the commutator estimates or the composition estimates in Lemma 2.1 below), such that the requirement for $a \in C^{\left[s_{\theta}\right]+1}$ and $b \in C^{\left[s_{u}\right]+1}$ can be relaxed, see, e.g., (1.18), (1.20). 


\section{Proofs}

Recall the Cauchy problem for the two-dimensional Boussinesq equations (1.1)-(1.3)

$$
\left\{\begin{array}{l}
\partial_{t} \theta+u \cdot \nabla \theta-\operatorname{div}(\kappa \nabla \theta)=0, \\
\partial_{t} u+u \cdot \nabla u-\operatorname{div}(\mu S u)+\nabla \Pi=\theta \overrightarrow{e_{2}}, \\
\operatorname{div} u=0, \\
\left.(\theta, u)\right|_{t=0}=\left(\theta_{0}, u_{0}\right),
\end{array}\right.
$$

where $\kappa=a(\theta) \in L^{\infty}\left(\mathbb{R} ;\left[\kappa_{*}, \kappa^{*}\right]\right), \mu=b(\theta) \in L^{\infty}\left(\mathbb{R} ;\left[\mu_{*}, \mu^{*}\right]\right)$ with $\kappa_{*}, \kappa^{*}, \mu_{*}, \mu^{*}$ four positive constants.

We are going to show the existence, uniqueness as well as the global-in-time regularity results in Theorem 1.2 in Sects. 2.1, 2.2 and 2.3, respectively.

Recall the definition of the $\|\cdot\|_{L_{T}^{q} X_{x}}$-norm in (1.12). The Gagliardo-Nirenberg's inequality

$$
\|f\|_{L_{T}^{4} L_{x}^{4}\left(\mathbb{R}^{2}\right)} \leq C\|f\|_{L_{T}^{2} L_{x}^{2}\left(\mathbb{R}^{2}\right)}^{\frac{1}{2}}\|\nabla f\|_{L_{T}^{2} L_{x}^{2}\left(\mathbb{R}^{2}\right)}^{\frac{1}{2}}
$$

as well as the equivalence relations between the norms

$$
\begin{aligned}
\|S u\|_{L_{x}^{2}\left(\mathbb{R}^{2}\right)}^{2} & =2\|\nabla u\|_{L_{x}^{2}\left(\mathbb{R}^{2}\right)}^{2} \text { if } \operatorname{div} u=0, \\
\|\Delta \eta\|_{L_{x}^{2}\left(\mathbb{R}^{2}\right)} & \sim\left\|\nabla^{2} \eta\right\|_{L_{x}^{2}\left(\mathbb{R}^{2}\right)}
\end{aligned}
$$

will be used freely in the proof.

\subsection{Existence of weak solutions if $\left(\theta_{0}, u_{0}\right) \in\left(L^{2}\left(\mathbb{R}^{2}\right)\right)^{3}$}

We will follow the standard procedure to show the existence of the weak solutions under the initial condition

$$
\left(\theta_{0}, u_{0}\right) \in L^{2}\left(\mathbb{R}^{2}\right) \times\left(L^{2}\left(\mathbb{R}^{2}\right)\right)^{2}
$$

namely

Step 1 We construct a sequence of approximate solutions, which satisfy the energy estimates uniformly. Step 2 We show the convergence of this approximate solution sequence to a weak solution and study the property of the weak solution.

We are going to sketch the proof and pay attention to the low-regularity assumptions.

\section{Step 1: Construction of approximate solutions with uniform bounds}

We use the Friedrich's method to construct a sequence of approximate solutions. We consider the following system of $\left(\theta_{n}, u_{n}\right)$

$$
\left\{\begin{array}{l}
\partial_{t} \theta_{n}+P_{n}\left(u_{n} \cdot \nabla \theta_{n}\right)-P_{n} \operatorname{div}\left(\kappa_{n} \nabla \theta_{n}\right)=0, \\
\partial_{t} u_{n}+P_{n} \mathbb{P}\left(u_{n} \cdot \nabla u_{n}\right)-P_{n} \mathbb{P} \operatorname{div}\left(\mu_{n} S u_{n}\right)=\mathbb{P}\left(\theta_{n} \overrightarrow{e_{2}}\right), \\
u_{n}(0, x)=P_{n} u_{0}(x), \quad \theta_{n}(0, x)=P_{n} \theta_{0}(x),
\end{array}\right.
$$

where $\kappa_{n}=a\left(\theta_{n}\right)$ and $\mu_{n}=b\left(\theta_{n}\right)$. The operator $P_{n}, n \in \mathbb{N}$, is the low-frequency cutoff operator which is defined as follows

$$
P_{n} f(x)=\mathcal{F}^{-1}\left(\nVdash_{B_{n}}(\xi) \mathcal{F} f(\xi)\right)(x),
$$

where $B_{n} \subset \mathbb{R}^{2}$ is the disk with center at 0 and radius $n$, and $\mathcal{F}, \mathcal{F}^{-1}$ are the standard Fourier and inverse Fourier transformations. The operator $\mathbb{P}$ in (2.4) denotes the Leray-Helmholtz projector on $\mathbb{R}^{2}$, which decomposes the tempered distributions $v \in \mathcal{S}^{\prime}\left(\mathbb{R}^{2} ; \mathbb{R}^{2}\right)$ into div-free and curl-free parts as follows

$$
v=\nabla^{\perp} V_{1}+\nabla V_{2},
$$


where

$$
\nabla^{\perp} V_{1}=-\nabla^{\perp}(-\Delta)^{-1} \nabla^{\perp} \cdot v=: \mathbb{P} v, \quad \nabla V_{2}=-\nabla(-\Delta)^{-1} \nabla \cdot v=(1-\mathbb{P}) v
$$

with $\nabla^{\perp}=\left(\partial_{x_{2}},-\partial_{x_{1}}\right)^{T}$. Notice that $\mathbb{P}$ maps $L^{p}\left(\mathbb{R}^{2} ; \mathbb{R}^{2}\right)$ into itself for any $p \in(1, \infty)$ and it is commutative with the projection operator $P_{n}$.

We define the Banach spaces $L_{n}^{2}$ and $L_{n}^{2, \sigma}$ as following

$$
\begin{aligned}
L_{n}^{2}\left(\mathbb{R}^{2}\right) & =\left\{f \in L^{2}\left(\mathbb{R}^{2}\right) \mid f=P_{n} f\right\}, \\
L_{n}^{2, \sigma}\left(\mathbb{R}^{2}\right) & =\left\{f \in\left(L_{n}^{2}\left(\mathbb{R}^{2}\right)\right)^{2} \mid \operatorname{div}_{x}(f)=0\right\} .
\end{aligned}
$$

The system (2.4) turns out to be an ordinary differential equation system in $L_{n}^{2}\left(\mathbb{R}^{2}\right) \times L_{n}^{2, \sigma}\left(\mathbb{R}^{2}\right)$. Indeed, the following estimates hold

$$
\begin{aligned}
& \left\|P_{n}\left(u_{n} \cdot \nabla \theta_{n}\right)-P_{n} \operatorname{div}\left(\kappa_{n} \nabla \theta_{n}\right)\right\|_{L_{x}^{2}} \leq C n^{3}\left(\left\|u_{n}\right\|_{L_{x}^{2}}+\kappa^{*}\right)\left\|\theta_{n}\right\|_{L_{x}^{2}}, \\
& \left\|P_{n} \mathbb{P}\left(u_{n} \cdot \nabla u_{n}\right)-P_{n} \mathbb{P} \operatorname{div}\left(\mu_{n} S u_{n}\right)\right\|_{L_{x}^{2}} \leq C n^{3}\left(\left\|u_{n}\right\|_{L_{x}^{2}}+\mu^{*}\right)\left\|u_{n}\right\|_{L_{x}^{2}} .
\end{aligned}
$$

Hence, for any $n \in \mathbb{N}$, there exists $T_{n}>0$ such that the system (2.4) has a solution $\left(\theta_{n}, u_{n}\right) \in$ $C\left(\left[0, T_{n}\right] ; L_{n}^{2}\left(\mathbb{R}^{2}\right)\right) \times C\left(\left[0, T_{n}\right] ; L_{n}^{2, \sigma}\left(\mathbb{R}^{2}\right)\right)$.

We take the $L^{2}\left(\mathbb{R}^{2}\right)$-inner product of Eq. $(2.4)_{1}$ and $\theta_{n}$ to derive

$$
\frac{1}{2} \frac{d}{d t} \int_{\mathbb{R}^{2}} \theta_{n}^{2}+\int_{\mathbb{R}^{2}} \kappa_{n}\left|\nabla \theta_{n}\right|^{2}=0 .
$$

Then, the following uniform estimate for $\left(\theta_{n}\right)$ holds

$$
\frac{1}{2}\left\|\theta_{n}\right\|_{L_{T}^{\infty} L_{x}^{2}}^{2}+\kappa_{*}\left\|\nabla \theta_{n}\right\|_{L_{T}^{2} L_{x}^{2}}^{2} d t \leq \frac{1}{2}\left\|P_{n} \theta_{0}\right\|_{L_{x}^{2}}^{2} \leq \frac{1}{2}\left\|\theta_{0}\right\|_{L_{x}^{2}}^{2}, \quad \forall T>0 .
$$

Similarly, we take the $L^{2}\left(\mathbb{R}^{2}\right)$-inner product of Eq. $(2.4)_{2}$ and $u_{n}$ to derive

$$
\frac{1}{2} \frac{\mathrm{d}}{\mathrm{d} t}\left\|u_{n}\right\|_{L_{x}^{2}}^{2}+\frac{1}{2}\left\|\mu_{n} S u_{n}\right\|_{L_{x}^{2}}^{2} \leq\left\|\theta_{n}\right\|_{L_{x}^{2}}\left\|u_{n}\right\|_{L_{x}^{2}} \leq \frac{1}{2}\left(T\left\|\theta_{n}\right\|_{L_{x}^{2}}^{2}+\frac{1}{T}\left\|u_{n}\right\|_{L_{x}^{2}}^{2}\right),
$$

for all positive times $T>0$, and thus by Gronwall's inequality we arrive at the following uniform estimate for $\left(u_{n}\right)$ (noticing $\left.\left\|S u_{n}\right\|_{L_{x}^{2}}^{2}=2\left\|\nabla u_{n}\right\|_{L_{x}^{2}}^{2}\right)$

$$
\frac{1}{2}\left\|u_{n}\right\|_{L_{T}^{\infty} L_{x}^{2}}^{2}+\mu_{*}\left\|\nabla u_{n}\right\|_{L_{T}^{2} L_{x}^{2}}^{2} \leq \frac{e}{2}\left(T\left\|\theta_{0}\right\|_{L_{x}^{2}}^{2}+\left\|u_{0}\right\|_{L_{x}^{2}}^{2}\right), \quad \forall T>0 .
$$

Thus, the approximate solutions $\left(\theta_{n}, u_{n}\right)$ exist for all positive times.

\section{Step 2: Passing to the limit}

By the above uniform bounds (2.6)-(2.7), there exists a subsequence, still denote by $\left(\theta_{n}, u_{n}\right)$, converging weakly to a limit $(\theta, u) \in L_{\mathrm{loc}}^{\infty}\left([0, \infty) ;\left(L_{x}^{2}\right)^{3}\right) \cap L_{\mathrm{loc}}^{2}\left([0, \infty) ;\left(H_{x}^{1}\right)^{3}\right)$ :

$$
\begin{aligned}
& \theta_{n} \stackrel{*}{\rightarrow} \theta \quad \text { in } L_{\mathrm{loc}}^{\infty}\left([0, \infty) ; L^{2}\left(\mathbb{R}^{2}\right)\right), \quad \nabla \theta_{n} \rightarrow \nabla \theta \quad \text { in } L_{\mathrm{loc}}^{2}\left([0, \infty) ;\left(L^{2}\left(\mathbb{R}^{2}\right)\right)^{2}\right), \\
& u_{n} \stackrel{*}{\rightarrow} u \quad \text { in } L_{\mathrm{loc}}^{\infty}\left([0, \infty) ;\left(L^{2}\left(\mathbb{R}^{2}\right)\right)^{2}\right), \quad \nabla u_{n} \rightarrow \nabla u \quad \text { in } L_{\mathrm{loc}}^{2}\left([0, \infty) ;\left(L^{2}\left(\mathbb{R}^{2}\right)\right)^{4}\right) .
\end{aligned}
$$

Since by the Gagliardo-Nirenberg's inequality $\left(\theta_{n}, u_{n}\right)$ is a bounded sequence in $L_{T}^{4} L_{x}^{4}$ for any $T>0$, the sequence of the time derivatives $\left(\partial_{t} \theta_{n}, \partial_{t} u_{n}\right)$ is bounded in $L_{T}^{2}\left(H_{x}^{-1}\right)$ [by use of the equations in $(2.4)$ ], and hence $\left\{\left(\theta_{n}, u_{n}\right)\right\}$ is relatively compact in $L_{T}^{p} L_{x}^{2}\left(B_{R}\right)$ for any fixed disk $B_{R} \subset \mathbb{R}^{2}$ and $p \in[1, \infty)$, which implies the pointwise convergence (up to a subsequence)

$$
\theta_{n} \rightarrow \theta, \quad u_{n} \rightarrow u \text { for almost every } t \in \mathbb{R}^{+}, \quad x \in \mathbb{R}^{2},
$$

as well as the convergence of the nonlinear terms (noticing, e.g., $u_{n} \varphi \rightarrow u \varphi$ in $L_{T}^{4} L_{x}^{4}$ for fixed $\varphi \in$ $\left.C_{c}^{\infty}\left((0, T) \times \mathbb{R}^{2}\right)\right)$

$$
u_{n} \theta_{n} \rightarrow u \theta, \quad u_{n} \otimes u_{n} \rightarrow u \otimes u \text { in } \mathcal{D}^{\prime}\left((0, T) \times \mathbb{R}^{2}\right) \text { and hence weakly in } L_{T}^{2} L_{x}^{2} .
$$


Consequently, $\kappa_{n}=a\left(\theta_{n}\right) \rightarrow \kappa=a(\theta)$ and $\mu_{n}=b\left(\theta_{n}\right) \rightarrow \mu=b(\theta)$ almost everywhere and

$$
\kappa_{n} \nabla \theta_{n} \rightarrow \kappa \nabla \theta, \quad \mu_{n} S u_{n} \rightarrow \mu S u \text { in } L_{T}^{2} L_{x}^{2} .
$$

Thus, the equation (noticing $P_{n} \rightarrow \mathrm{Id}$ as an operator from $H^{s}\left(\mathbb{R}^{2}\right)$ to itself)

$$
\partial_{t} \theta+\operatorname{div}(u \theta)-\operatorname{div}(\kappa \nabla \theta)=0 \text { holds in } L_{\text {loc }}^{2}\left((0, \infty) ; H_{x}^{-1}\left(\mathbb{R}^{2}\right)\right),
$$

and we can test it by $\theta \in L_{\text {loc }}^{2}\left([0, \infty) ; H_{x}^{1}\right)$ to arrive at the energy equality (1.8) for $\theta$, such that $\left.\theta\right|_{t=0}=\theta_{0}$ and $\theta \in C\left([0, \infty) ; L_{x}^{2}\right)$ hold true.

Similarly, the equation

$$
\partial_{t} u+\mathbb{P} \operatorname{div}(u \otimes u-\mu S u)=\mathbb{P}\left(\theta \vec{e}_{2}\right) \text { holds in } L_{\mathrm{loc}}^{2}\left((0, \infty) ;\left(H_{x}^{-1}\left(\mathbb{R}^{2}\right)\right)^{2}\right),
$$

and we can test it by the divergence-free velocity field $u \in L_{\text {loc }}^{2}\left((0, \infty) ;\left(H_{x}^{1}\left(\mathbb{R}^{2}\right)\right)^{2}\right)$ to arrive at the energy equality (1.10), which implies $u \in C\left([0, \infty) ;\left(L_{x}^{2}\left(\mathbb{R}^{2}\right)\right)^{2}\right)$ and $\left.u\right|_{t=0}=u_{0}$. We take the solution $\Pi$ of the Poisson equation

$$
\Delta \Pi=\operatorname{div}(1-\mathbb{P})\left(\theta \vec{e}_{2}-\operatorname{div}(u \otimes u-\mu S u)\right)
$$

under the renormalization condition $\int_{B_{1}} \Pi \mathrm{d} x=0$, such that

$$
\nabla \Pi=(1-\mathbb{P})\left(\theta \vec{e}_{2}-\operatorname{div}(u \otimes u-\mu S u)\right) \in L_{\mathrm{loc}}^{2}\left((0, \infty) ;\left(H_{x}^{-1}\left(\mathbb{R}^{2}\right)\right)^{2}\right),
$$

and the equation $(1.11)$ holds in $L_{\mathrm{loc}}^{2}\left((0, \infty) ;\left(H_{x}^{-1}\left(\mathbb{R}^{2}\right)\right)^{2}\right)$.

\subsection{Energy estimates and uniqueness of the weak solutions if $\left(\theta_{0}, u_{0}\right) \in H^{1}\left(\mathbb{R}^{2}\right) \times\left(L^{2}\left(\mathbb{R}^{2}\right)\right)^{2}$}

We first introduce a scalar function $\eta$, which is given in terms of the temperature function as follows (recalling now $\kappa=a(\theta) \in C_{b}^{2}\left(\mathbb{R} ;\left[\kappa_{*}, \kappa^{*}\right]\right)$ )

$$
\eta=A(\theta), \text { with } A(z):=\int_{0}^{z} a(\alpha) d \alpha \text { the primitive function of } a .
$$

As $A^{\prime}(\theta)=a(\theta) \geq \kappa_{*}>0$, the function $A$ is invertible and we can write

$$
\theta=A^{-1}(\eta)
$$

where $\left(A^{-1}\right)^{\prime}(\eta)=\frac{1}{a\left(A^{-1}(\eta)\right)} \leq \frac{1}{\kappa^{*}}$. We have the following equivalence relations ${ }^{3}$

$$
\begin{aligned}
& \kappa_{*}\|\theta\|_{L_{x}^{2}} \leq\|\eta\|_{L_{x}^{2}} \leq \kappa^{*}\|\theta\|_{L_{x}^{2}}, \\
& \kappa_{*}\|\nabla \theta\|_{L_{x}^{2}} \leq\|\nabla \eta\|_{L_{x}^{2}}=\|a(\theta) \nabla \theta\|_{L_{x}^{2}} \leq \kappa^{*}\|\nabla \theta\|_{L_{x}^{2}}, \\
& \kappa_{*}\left\|\partial_{t} \theta\right\|_{L_{x}^{2}} \leq\left\|\partial_{t} \eta\right\|_{L_{x}^{2}}=\left\|a(\theta) \partial_{t} \theta\right\|_{L_{x}^{2}} \leq \kappa^{*}\left\|\partial_{t} \theta\right\|_{L_{x}^{2}}, \\
& \left\|\nabla^{2} \eta\right\|_{L_{x}^{2}} \leq\|a\|_{\text {Lip }}\|\nabla \theta\|_{L_{x}^{4}}^{2}+\kappa^{*}\left\|\nabla^{2} \theta\right\|_{L_{x}^{2}} \leq\left(C\|a\|_{\text {Lip }}\|\nabla \theta\|_{L_{x}^{2}}+\kappa^{*}\right)\left\|\nabla^{2} \theta\right\|_{L_{x}^{2}}, \\
& \left\|\nabla^{2} \theta\right\|_{L_{x}^{2}} \leq \frac{\|a\|_{\text {Lip }}}{\kappa_{*}^{3}}\|\nabla \eta\|_{L_{x}^{4}}^{2}+\frac{1}{\kappa_{*}}\left\|\nabla^{2} \eta\right\|_{L_{x}^{2}} \leq\left(C \frac{\|a\|_{\text {Lip }}}{\kappa_{*}^{3}}\|\nabla \eta\|_{L_{x}^{2}}+\frac{1}{\kappa_{*}}\right)\left\|\nabla^{2} \eta\right\|_{L_{x}^{2}} .
\end{aligned}
$$

\footnotetext{
${ }^{3}$ We can easily compute

$$
\begin{aligned}
& \nabla \eta=a(\theta) \nabla \theta, \quad \nabla \theta=\frac{1}{a\left(A^{-1}(\eta)\right)} \nabla \eta, \\
& \nabla^{2} \eta=a^{\prime}(\theta) \nabla \theta \otimes \nabla \theta+a(\theta) \nabla^{2} \theta, \quad \nabla^{2} \theta=-\frac{a^{\prime}\left(A^{-1}(\eta)\right)}{a^{3}\left(A^{-1}(\eta)\right)} \nabla \eta \otimes \nabla \eta+\frac{1}{a\left(A^{-1}(\eta)\right)} \nabla^{2} \eta .
\end{aligned}
$$
}


That is,

$$
\theta(t, \cdot) \in H_{x}^{k}\left(\mathbb{R}^{2}\right) \Leftrightarrow \eta(t, \cdot) \in H_{x}^{k}\left(\mathbb{R}^{2}\right), \quad k=0,1,2 .
$$

Let $(\theta, u) \in C\left([0, \infty) ;\left(L^{2}\left(\mathbb{R}^{2}\right)\right)^{3}\right) \cap L_{\text {loc }}^{2}\left([0, \infty) ;\left(H^{1}\left(\mathbb{R}^{2}\right)\right)^{3}\right)$ be a weak solution of the Cauchy problem (2.1) in the sense of Definition 1.1 with

$$
\partial_{t} \theta+u \cdot \nabla \theta-\operatorname{div}(\kappa \nabla \theta)=0 \text { holding in } L_{\text {loc }}^{2}\left([0, \infty) ; H_{x}^{-1}\left(\mathbb{R}^{2}\right)\right) .
$$

Since $Y:=L_{t, x}^{\infty}\left([0, \infty) \times \mathbb{R}^{2}\right) \cap L_{\text {loc }}^{2}\left([0, \infty) ; H_{x}^{1}\left(\mathbb{R}^{2}\right)\right)$ is an algebra (in the sense that the product of any two elements in $Y$ still belongs to $Y$ ), we can multiply the above $\theta$-equation by $\kappa=a(\theta)$ (with $a(\theta)-a(0) \in Y)$, to arrive at the parabolic equation for $\eta=A(\theta) \in C\left([0, \infty) ; L^{2}\left(\mathbb{R}^{2}\right)\right) \cap L_{\text {loc }}^{2}\left([0, \infty) ; H^{1}\left(\mathbb{R}^{2}\right)\right)$ :

$$
\partial_{t} \eta+u \cdot \nabla \eta-\kappa \Delta \eta=0 \text { holding in the dual space } Y^{\prime} .
$$

We are going to derive the $H^{1}$-Estimate for $\eta$ (and hence for $\theta^{4}$ ) as well as the $L^{2}$-Estimate for $u$ first. Then, we will show the uniqueness result of the weak solutions by considering the difference of two possible weak solutions. The procedure is standard (see, e.g., Sect. 2 [29]) and we are going to sketch the proof.

$H^{1} \times L^{2}$-Estimate for $(\theta, u)$

By virtue of the energy equalities (1.8) and (1.10) and the derivation of the uniform estimates (2.6) and (2.7), we have the $L^{2}$-Estimate

$$
\|\theta\|_{L_{T}^{\infty} L_{x}^{2}}^{2}+\|\nabla \theta\|_{L_{T}^{2} L_{x}^{2}}^{2} \leq C\left(\kappa_{*}\right)\left\|\theta_{0}\right\|_{L^{2}}^{2},
$$

and the $L^{2}$-Estimate (1.13) for $u$. By Gagliardo-Nirenberg's inequality (2.2) it holds

$$
\|u\|_{L_{T}^{4} L_{x}^{4}} \leq C\left(\mu_{*}\right)\left(\sqrt{T}\left\|\theta_{0}\right\|_{L^{2}}+\left\|u_{0}\right\|_{L^{2}}\right) .
$$

We assume a priori that the function $\eta$ is smooth and decay sufficiently fast at infinity. We test the $\eta$-equation (2.15) by $\Delta \eta$ to derive by integration by parts that

$$
\frac{1}{2} \frac{\mathrm{d}}{\mathrm{d} t} \int_{\mathbb{R}^{2}}|\nabla \eta|^{2} \mathrm{~d} x+\int_{\mathbb{R}^{2}} \kappa|\Delta \eta|^{2} \mathrm{~d} x=\int_{\mathbb{R}^{2}} u \cdot \nabla \eta \Delta \eta \mathrm{d} x \leq\|u\|_{L_{x}^{4}}\|\nabla \eta\|_{L_{x}^{4}}\|\Delta \eta\|_{L_{x}^{2}} .
$$

By Gagliardo-Nirenberg's inequality (2.2), the equivalence $\|\Delta \eta\|_{L_{x}^{2}\left(\mathbb{R}^{2}\right)} \sim\left\|\nabla^{2} \eta\right\|_{L_{x}^{2}\left(\mathbb{R}^{2}\right)}$ and Young's inequality we arrive at

$$
\frac{1}{2} \frac{\mathrm{d}}{\mathrm{d} t} \int_{\mathbb{R}^{2}}|\nabla \eta|^{2} \mathrm{~d} x+\frac{\kappa_{*}}{2} \int_{\mathbb{R}^{2}}|\Delta \eta|^{2} \mathrm{~d} x \leq C\left(\kappa_{*}\right)\|u\|_{L_{x}^{4}}^{4}\|\nabla \eta\|_{L_{x}^{2}}^{2} .
$$

Gronwall's inequality gives

$$
\|\nabla \eta(T)\|_{L_{x}^{2}}^{2}+\left\|\nabla^{2} \eta\right\|_{L_{T}^{2} L_{x}^{2}}^{2} \leq C\left(\kappa_{*}\right)\left\|\nabla \eta_{0}\right\|_{L_{x}^{2}}^{2} \exp \left(C\left(\kappa_{*}\right)\|u\|_{L_{T}^{4} L_{x}^{4}}^{4}\right)
$$

for any positive time $T>0$. Thus by the $\eta$-equation

$$
\begin{aligned}
\left\|\partial_{t} \eta\right\|_{L_{T}^{2} L_{x}^{2}} & =\|u \cdot \nabla \eta-\kappa \Delta \eta\|_{L_{T}^{2} L_{x}^{2}} \leq\|u\|_{L_{T}^{4} L_{x}^{4}}\|\nabla \eta\|_{L_{T}^{4} L_{x}^{4}}+\kappa^{*}\|\Delta \eta\|_{L_{T}^{2} L_{x}^{2}} \\
& \leq C\left(\kappa_{*}, \kappa^{*}\right)\left\|\nabla \eta_{0}\right\|_{L_{x}^{2}} \exp \left(C\left(\kappa_{*}\right)\|u\|_{L_{T}^{4} L_{x}^{4}}^{4}\right) .
\end{aligned}
$$

By virtue of the equivalence relation (2.12):

$$
\|\nabla \theta\|_{L_{T}^{\infty} L_{x}^{2}}^{2}+\left\|\nabla^{2} \theta\right\|_{L_{T}^{2} L_{x}^{2}}^{2} \leq C\left(\kappa_{*},\|a\|_{\text {Lip }}\right)\left(\|\nabla \eta\|_{L_{T}^{\infty} L_{x}^{2}}^{2}+\left(1+\|\nabla \eta\|_{L_{T}^{\infty} L_{x}^{2}}^{2}\right)\left\|\nabla^{2} \eta\right\|_{L_{T}^{2} L_{x}^{2}}^{2}\right)
$$

\footnotetext{
${ }^{4}$ The introduction of the $\eta$-function makes the derivation of the $H^{1}$-Estimate for $\theta$ straightforward (and possible).
} 
and (2.16)-(2.17), we have the a priori $H^{1}$-Estimate (1.14) for $\theta$ :

$$
\begin{aligned}
& \|\theta\|_{L_{T}^{\infty} H_{x}^{1}}^{2}+\|\nabla \theta\|_{L_{T}^{2} H_{x}^{1}}^{2}+\left\|\partial_{t} \theta\right\|_{L_{T}^{2} L_{x}^{2}}^{2} \\
& \leq C\left(\kappa_{*},\|a\|_{\text {Lip }}, \kappa^{*}\right)\left\|\theta_{0}\right\|_{H^{1}}^{2}\left(1+\left\|\nabla \theta_{0}\right\|_{L^{2}}^{2}\right) \exp \left(C\left(\kappa_{*}\right)\|u\|_{L_{T}^{4} L_{x}^{4}}^{4}\right) .
\end{aligned}
$$

Therefore, both the parabolic equations (2.14) and (2.15) for $\theta$ and $\eta$ hold in $L_{\text {loc }}^{2}\left([0, \infty) ; L^{2}\left(\mathbb{R}^{2}\right)\right)$. A standard density argument ensures the $H^{1}$-Estimate $(1.14)$ for $\theta$, and hence $\theta \in C\left([0, \infty) ; H_{x}^{1}\left(\mathbb{R}^{2}\right)\right)$.

\section{Proof of the uniqueness}

Let $\left(\theta_{1}, u_{1}, \Pi_{1}\right)$ and $\left(\theta_{2}, u_{2}, \Pi_{2}\right)$ be two weak solutions of the Cauchy problem (2.1) with the same initial data $\left(\theta_{0}, u_{0}\right) \in H^{1}\left(\mathbb{R}^{2}\right) \times\left(L^{2}\left(\mathbb{R}^{2}\right)\right)^{2}$, which satisfy the energy estimates (1.13)-(1.14). Recall (2.10) for the definition of the function $A$, and we set

$$
\eta_{1}=A\left(\theta_{1}\right), \quad \eta_{2}=A\left(\theta_{2}\right) .
$$

We consider the difference

$$
(\dot{\eta}, \dot{u}, \nabla \dot{\Pi})=\left(\eta_{1}-\eta_{2}, u_{1}-u_{2}, \nabla \Pi_{1}-\nabla \Pi_{2}\right)
$$

which lies in

$$
\begin{aligned}
& \left(C\left([0, \infty) ; H^{1}\left(\mathbb{R}^{2}\right)\right) \cap L_{\mathrm{loc}}^{2}\left([0, \infty) ; H^{2}\left(\mathbb{R}^{2}\right)\right)\right) \\
& \left.\quad \times\left(C\left([0, \infty) ;\left(L^{2}\left(\mathbb{R}^{2}\right)\right)^{2}\right) \cap L_{\mathrm{loc}}^{2}\left([0, \infty) ;\left(H^{1}\left(\mathbb{R}^{2}\right)\right)^{2}\right)\right) \times L_{\mathrm{loc}}^{2}\left([0, \infty) ;\left(H^{-1}\left(\mathbb{R}^{2}\right)\right)^{2}\right)\right) .
\end{aligned}
$$

It satisfies the following Cauchy problem

$$
\left\{\begin{array}{l}
\partial_{t} \dot{\eta}+u_{1} \cdot \nabla \dot{\eta}-\kappa_{1} \Delta \dot{\eta}=\dot{\kappa} \Delta \eta_{2}-\dot{u} \cdot \nabla \eta_{2} \\
\partial_{t} \dot{u}+u_{1} \cdot \nabla \dot{u}-\operatorname{div}\left(\mu_{1} S \dot{u}\right)+\nabla \dot{\Pi}=\dot{\theta} \overrightarrow{e_{2}}-\dot{u} \cdot \nabla u_{2}+\operatorname{div}\left(\dot{\mu} S u_{2}\right), \\
\operatorname{div} \dot{u}=0 \\
\left(\dot{\eta}_{0}, \dot{u}_{0}\right)=(0,0)
\end{array}\right.
$$

where

$$
\kappa_{1}=a\left(\theta_{1}\right), \mu_{1}=b\left(\theta_{1}\right), \dot{\theta}=\theta_{1}-\theta_{2}, \dot{\kappa}=a\left(\theta_{1}\right)-a\left(\theta_{2}\right), \dot{\mu}=b\left(\theta_{1}\right)-b\left(\theta_{2}\right) .
$$

Similarly as in (2.12) we have the following equivalence relationships

$$
\begin{aligned}
\kappa_{*}\|\dot{\theta}\|_{L_{x}^{2}} & \leq\|\dot{\eta}\|_{L_{x}^{2}} \leq \kappa^{*}\|\dot{\theta}\|_{L_{x}^{2}}, \\
\|\nabla \dot{\eta}\|_{L_{x}^{2}} & \leq\|a\|_{\text {Lip }}\left\|\nabla \theta_{1}\right\|_{L_{x}^{4}}\|\dot{\theta}\|_{L_{x}^{4}}+\kappa^{*}\|\nabla \dot{\theta}\|_{L_{x}^{2}}, \\
\|\nabla \dot{\theta}\|_{L_{x}^{2}} & \leq \frac{\|a\|_{\text {Lip }}}{\kappa_{*}^{3}}\left\|\nabla \eta_{1}\right\|_{L_{x}^{4}}\|\dot{\eta}\|_{L_{x}^{4}}+\frac{1}{\kappa_{*}}\|\nabla \dot{\eta}\|_{L_{x}^{2}},
\end{aligned}
$$

and correspondingly we have

$$
\begin{aligned}
\|(\dot{\kappa}, \dot{\mu})\|_{H_{x}^{1}} & \leq C\left(\|(a, b)\|_{\text {Lip }},\left\|\left(a^{\prime}, b^{\prime}\right)\right\|_{\text {Lip }}, \kappa_{*}\right)\left(\left\|\nabla \eta_{1}\right\|_{L_{x}^{4}}\|\dot{\eta}\|_{L_{x}^{4}}+\|\dot{\eta}\|_{H_{x}^{1}}\right) \\
& \leq C\left(\|(a, b)\|_{\text {Lip }},\left\|\left(a^{\prime}, b^{\prime}\right)\right\|_{\text {Lip }}, \kappa_{*}\right)\left(1+\left\|\nabla \eta_{1}\right\|_{L_{x}^{4}}\right)\|\dot{\eta}\|_{H_{x}^{1}} .
\end{aligned}
$$

We are going to sketch the derivation of the $H^{1} \times L^{2}$-Estimate for $(\dot{\eta}, \dot{u})$.

(i) $L^{2}$ estimate of $\dot{\eta}$. We take the $L^{2}\left(\mathbb{R}^{2}\right)$-inner product between $(2.19)_{1}$ and $\dot{\eta}$ to derive

$$
\frac{1}{2} \frac{\mathrm{d}}{\mathrm{d} t} \int_{\mathbb{R}^{2}}|\dot{\eta}|^{2}+\int_{\mathbb{R}^{2}} \kappa_{1}|\nabla \dot{\eta}|^{2} \leq \int_{\mathbb{R}^{2}}\left|\dot{\eta} \nabla \kappa_{1} \cdot \nabla \dot{\eta}\right|+\left|\dot{\eta} \dot{u} \cdot \nabla \eta_{2}\right|+\left|\dot{\kappa} \Delta \eta_{2} \dot{\eta}\right| .
$$

The right hand side can be bounded by

$$
\begin{aligned}
& \|\dot{\eta}\|_{L_{x}^{4}}\left\|\nabla \kappa_{1}\right\|_{L_{x}^{4}}\|\nabla \dot{\eta}\|_{L_{x}^{2}}+\left\|\nabla \eta_{2}\right\|_{L_{x}^{2}}\|\dot{u}\|_{L_{x}^{4}}\|\dot{\eta}\|_{L_{x}^{4}}+\left\|\Delta \eta_{2}\right\|_{L_{x}^{2}}\|\dot{\kappa}\|_{L_{x}^{4}}\|\dot{\eta}\|_{L_{x}^{4}} \\
& \quad \leq C\left(\|a\|_{L_{i p}}\right)\left(\left\|\nabla \theta_{1}\right\|_{L_{x}^{4}}\|\dot{\eta}\|_{L_{x}^{2}}^{\frac{1}{2}}\|\nabla \dot{\eta}\|_{L_{x}^{2}}^{\frac{3}{2}}+\left\|\nabla \eta_{2}\right\|_{L_{x}^{2}}\|\dot{u}\|_{L_{x}^{2}}^{\frac{1}{2}}\|\nabla \dot{u}\|_{L_{x}^{2}}^{\frac{1}{2}}\|\dot{\eta}\|_{L_{x}^{2}}^{\frac{1}{2}}\|\nabla \dot{\eta}\|_{L_{x}^{2}}^{\frac{1}{2}}\right.
\end{aligned}
$$




$$
\begin{aligned}
& \left.+\left\|\Delta \eta_{2}\right\|_{L_{x}^{2}}\|\dot{\theta}\|_{L_{x}^{2}}^{\frac{1}{2}}\|\nabla \dot{\theta}\|_{L_{x}^{2}}^{\frac{1}{2}}\|\dot{\eta}\|_{L_{x}^{2}}^{\frac{1}{2}}\|\nabla \dot{\eta}\|_{L_{x}^{2}}^{\frac{1}{2}}\right) \\
& \leq \frac{\kappa_{*}}{2}\|\nabla \dot{\eta}\|_{L_{x}^{2}}^{2}+\frac{\mu_{*}}{4}\|\nabla \dot{u}\|_{L_{x}^{2}}^{2} \\
& +C\left(\|a\|_{L_{\text {Lip }}}, \kappa_{*}, \mu_{*}\right)\left(\left\|\nabla \theta_{1}\right\|_{L_{x}^{4}}^{4}+\left\|\nabla \eta_{2}\right\|_{L_{x}^{2}}^{2}+\left\|\Delta \eta_{2}\right\|_{L_{x}^{2}}^{2}\right) \times\left(\|\dot{\eta}\|_{L_{x}^{2}}^{2}+\|\dot{u}\|_{L_{x}^{2}}^{2}\right) .
\end{aligned}
$$

(ii) $L^{2}$ estimate of $\dot{u}$.

We take the $L^{2}$ inner product of the equation $(2.19)_{2}$ and $\dot{u}$ to derive

$$
\frac{1}{2} \frac{\mathrm{d}}{\mathrm{d} t} \int_{\mathbb{R}^{2}}|\dot{u}|^{2}+\frac{1}{2} \int_{\mathbb{R}^{2}} \mu_{1}|S \dot{u}|^{2} \leq \int_{\mathbb{R}^{2}}|\dot{\theta} \dot{u}|+|\dot{u}|^{2}\left|\nabla u_{2}\right|+\left|\dot{\mu} S u_{2}: \nabla \dot{u}\right| .
$$

The right hand side can be bounded by

$$
\|\dot{u}\|_{L_{x}^{2}}\|\dot{\theta}\|_{L_{x}^{2}}+\|\dot{u}\|_{L_{x}^{4}}^{2}\left\|\nabla u_{2}\right\|_{L_{x}^{2}}+\|\dot{\mu}\|_{H_{x}^{1}}\left\|S u_{2}: \nabla \dot{u}\right\|_{H_{x}^{-1}}
$$

which, by use of the Sobolev embedding $L^{1}\left(\mathbb{R}^{2}\right) \hookrightarrow H^{-1}\left(\mathbb{R}^{2}\right)$, is bounded by

$$
\begin{aligned}
& \frac{\mu_{*}}{4}\|\nabla \dot{u}\|_{L_{x}^{2}}^{2}+C\left(\|a\|_{L i p}, \kappa_{*}, \mu_{*}\right)\left(1+\left\|\nabla u_{2}\right\|_{L_{x}^{2}}^{2}\right)\left(\|\dot{u}\|_{L_{x}^{2}}^{2}+\|\dot{\eta}\|_{L_{x}^{2}}^{2}\right) \\
& \quad+C\left(\mu_{*}\right)\left\|\nabla u_{2}\right\|_{L_{x}^{2}}^{2}\|\dot{\mu}\|_{H_{x}^{1}}^{2} .
\end{aligned}
$$

(iii) $L^{2}$ estimate of $\nabla \dot{\eta}$.

We take the $L^{2}$ inner product of Eq. (2.19) ${ }_{1} a n d \Delta \dot{\eta}$ to derive

$$
\frac{1}{2} \frac{\mathrm{d}}{\mathrm{d} t} \int_{\mathbb{R}^{2}}|\nabla \dot{\eta}|^{2}+\int_{\mathbb{R}^{2}} \kappa_{1}|\Delta \dot{\eta}|^{2} \leq \int_{\mathbb{R}^{2}}\left|u_{1} \cdot \nabla \dot{\eta} \Delta \dot{\eta}\right|+\left|\dot{u} \cdot \nabla \eta_{2} \Delta \dot{\eta}\right|+\left|\dot{\kappa} \Delta \eta_{2} \Delta \dot{\eta}\right|
$$

By $L^{1}\left(\mathbb{R}^{2}\right) \hookrightarrow H^{-1}\left(\mathbb{R}^{2}\right)$ again, the right hand side is bounded similarly by

$$
\begin{aligned}
& \frac{\kappa_{*}}{2}\|\Delta \dot{\eta}\|_{L_{x}^{2}}^{2}+\frac{\mu_{*}}{4}\|\nabla \dot{u}\|_{L_{x}^{2}}^{2}+C\left(\kappa_{*}, \mu_{*}\right)\left(\left\|u_{1}\right\|_{L_{x}^{4}}^{4}+\left\|\nabla \eta_{2}\right\|_{L_{x}^{4}}^{4}\right)\left(\|\dot{u}\|_{L_{x}^{2}}^{2}+\|\nabla \dot{\eta}\|_{L_{x}^{2}}^{2}\right) \\
& \quad+C\left(\kappa_{*}\right)\left\|\Delta \eta_{2}\right\|_{L_{x}^{2}}^{2}\|\dot{\kappa}\|_{H_{x}^{1}}^{2} .
\end{aligned}
$$

To conclude, by virtue of the above estimates and (2.21), we have the following inequality

$$
\begin{aligned}
& \frac{\mathrm{d}}{\mathrm{d} t}\left(\|\dot{\eta}\|_{H_{x}^{1}}^{2}+\|\dot{u}\|_{L_{x}^{2}}^{2}\right)+\|\nabla \dot{u}\|_{L_{x}^{2}}^{2}+\|\nabla \dot{\eta}\|_{H_{x}^{1}}^{2} \\
& \quad \leq C\left(\|(a, b)\|_{\text {Lip }},\left\|\left(a^{\prime}, b^{\prime}\right)\right\|_{L_{i p}}, \kappa_{*}, \mu_{*}\right) B(t)\left(\|\dot{u}\|_{L_{x}^{2}}^{2}+\|\dot{\eta}\|_{H_{x}^{1}}^{2}\right),
\end{aligned}
$$

where

$$
\begin{aligned}
B(t)= & \left(\left\|\nabla \theta_{1}\right\|_{L_{x}^{4}}^{4}+\left\|\nabla \eta_{2}\right\|_{L_{x}^{2}}^{2}+\left\|\Delta \eta_{2}\right\|_{L_{x}^{2}}^{2}+1+\left\|\nabla u_{2}\right\|_{L_{x}^{2}}^{2}+\left\|u_{1}\right\|_{L_{x}^{4}}^{4}+\left\|\nabla \eta_{2}\right\|_{L_{x}^{4}}^{4}\right) \\
& \times\left(1+\left\|\nabla \eta_{1}\right\|_{L_{x}^{4}}\right) \in L_{\mathrm{loc}}^{1}([0, \infty)) .
\end{aligned}
$$

Gronwall's inequality implies then $\dot{\eta}=0$ and $\dot{u}=0$. The uniqueness of the weak solutions follows.

\subsection{Propagation of the general $H^{s}$-regularities}

After the derivation of the a priori $H_{x}^{s}, s \in(0,2)$-Estimate for a general linear parabolic equation in Sect. 2.3.1, we are going to derive the precise $H_{x}^{s}$-Estimates (1.17)-(1.24) in Remark 1.4 in the subsequent subsections:

- In Sect. 2.3.2 the global-in-time $H_{x}^{s}\left(\mathbb{R}^{2}\right) \times\left(L_{x}^{2}\left(\mathbb{R}^{2}\right)\right)^{2}, s \in(1,2)$-regularities [i.e., (1.17)] will be established, where the endpoint case $s=2$ [i.e., (1.18)] will be treated separately.

- In Sect. 2.3.3, the global-in-time $H_{x}^{1}\left(\mathbb{R}^{2}\right) \times\left(H_{x}^{s}\left(\mathbb{R}^{2}\right)\right)^{2}, s \in(0,2)$-regularities [i.e., (1.19)] will be established, where the endpoint case $s=2$ [i.e., (1.20)] will be treated separately. 
- In Sect. 2.3.4 the global-in-time $H_{x}^{s}\left(\mathbb{R}^{2}\right) \times\left(H_{x}^{s-2}\left(\mathbb{R}^{2}\right)\right)^{2}$ [i.e., $\left.(1.21)-(1.22)\right]$ and $H_{x}^{s-1} \times\left(H_{x}^{s}\left(\mathbb{R}^{2}\right)\right)^{2}$, $s>2$-regularities [i.e., (1.23)-(1.24)] will be established, respectively.

As far as the borderline estimates (1.17)-(1.24) are established, the global-in-time regularity (1.16) follows immediately.

For readers' convenience, we recall here briefly the Littlewood-Paley dyadic decomposition and the definition of the $H^{s}\left(\mathbb{R}^{n}\right)$-norms (see, e.g., Chapter 2 in the book [4] for more details). We fix a nonincreasing radial function $\chi \in C_{c}^{\infty}\left(B_{\frac{4}{3}}\right)$ with $\chi(x)=1$ for $x \in B_{1}$, where $B_{r} \subset \mathbb{R}^{n}$ denotes the ball centered at 0 with radius $r$. We define the function $\varphi(\xi)=\chi\left(\frac{\xi}{2}\right)-\chi(\xi)$ and $\varphi_{j}(\xi)=\varphi\left(2^{-j} \xi\right)$ with $j \geq 0$. We do the Littlewood-Paley decomposition in the following way

$$
g=\Delta_{-1} g+\sum_{j \geq 0} \Delta_{j} g
$$

where

$$
\mathcal{F}\left(\Delta_{-1} g\right)(\xi)=\chi(\xi) \mathcal{F}(g)(\xi), \quad \mathcal{F}\left(\Delta_{j} g\right)(\xi)=\varphi_{j}(\xi) \mathcal{F}(g)(\xi), \quad j \geq 0,
$$

and $\mathcal{F}$ denotes the Fourier transform. We have the following Bernstein's inequalities for some universal constant $C$ (depending only on $n$ )

$$
\begin{aligned}
& \left\|\Delta_{-1} g\right\|_{L^{2}\left(\mathbb{R}^{n}\right)} \leq C\|g\|_{L^{2}\left(\mathbb{R}^{n}\right)}, \\
& C^{-1} 2^{j}\left\|\Delta_{j} g\right\|_{L^{2}\left(\mathbb{R}^{n}\right)} \leq\left\|\nabla\left(\Delta_{j} g\right)\right\|_{L^{2}\left(\mathbb{R}^{n}\right)} \leq C 2^{j}\left\|\Delta_{j} g\right\|_{L^{2}\left(\mathbb{R}^{n}\right)}, \quad \forall j \geq 0 .
\end{aligned}
$$

Let $s \geq 0$ and $p, r \geq 1$. We define the nonhomogeneous Besov spaces $B_{p, r}^{s}\left(\mathbb{R}^{n}\right)$ as the spaces consisting of all tempered distributions $g \in \mathcal{S}^{\prime}\left(\mathbb{R}^{n}\right)$ satisfying

$$
\|g\|_{B_{p, r}^{s}\left(\mathbb{R}^{n}\right)}=\left\|\left(2^{j s}\left\|\Delta_{j} g\right\|_{L^{p}\left(\mathbb{R}^{n}\right)}\right)_{j \geq-1}\right\|_{l^{r}}<\infty .
$$

The inhomogeneous Sobolev spaces $H^{s}\left(\mathbb{R}^{n}\right)=B_{2,2}^{s}\left(\mathbb{R}^{n}\right)$ can be defined by

$$
H^{s}\left(\mathbb{R}^{n}\right)=\left\{g \in \mathcal{S}^{\prime}\left(\mathbb{R}^{n}\right) \mid\|g\|_{H^{s}\left(\mathbb{R}^{n}\right)}=\left(\int_{\mathbb{R}^{n}}\left(1+|\xi|^{2}\right)^{\frac{s}{2}}|\mathcal{F}(g)(\xi)|^{2} d \xi\right)^{1 / 2}<\infty\right\},
$$

where the $H^{s}\left(\mathbb{R}^{n}\right)$-norm reads in terms of Littlewood-Paley decomposition as follows

$$
\|g\|_{H^{s}\left(\mathbb{R}^{n}\right)} \sim\|g\|_{L^{2}\left(\mathbb{R}^{n}\right)}+\left(\sum_{j \geq 0} 2^{2 j s}\left\|\Delta_{j} g\right\|_{L^{2}\left(\mathbb{R}^{n}\right)}^{2}\right)^{\frac{1}{2}} .
$$

It is straightforward to derive the following interpolation inequality

$$
\|u\|_{H^{t_{\sigma}}} \leq C\|u\|_{H^{t_{0}}}^{1-\sigma}\|u\|_{H^{t_{1}}}^{\sigma}, \text { where } t_{\sigma}=(1-\sigma) t_{0}+\sigma t_{1}, \sigma \in[0,1]
$$

We are going to use the following known estimates to control the nonlinear terms in the Boussinesq system (1.1).

Lemma 2.1. We have the following commutator, product, and composition estimates.

(1) $\left[12\right.$, Proposition 2.4] In the low regularity regime where $(s, \nu) \in \mathbb{R}^{2}$ satisfy

$$
-1<s<\nu+1, \quad \text { and }-1<\nu<1,
$$

the following commutator estimate holds true (in $\mathbb{R}^{2}$ ):

$$
\left\|\left(2^{j s}\left\|\left[\phi, \Delta_{j}\right] \nabla \psi\right\|_{L^{2}\left(\mathbb{R}^{2}\right)}\right)_{j \geq 1}\right\|_{l^{1}} \leq C\|\nabla \phi\|_{H^{\nu}\left(\mathbb{R}^{2}\right)}\|\nabla \psi\|_{H^{s-\nu}\left(\mathbb{R}^{2}\right)},
$$

where $C$ is a constant depending only on $s, \nu$. 
(2) [4, Lemma 2.100] For any $s>0$, the following commutator estimate holds true

$$
\begin{aligned}
& \left\|\left(2^{j s}\left\|\left[\phi, \Delta_{j}\right] \nabla \psi\right\|_{L^{2}\left(\mathbb{R}^{n}\right)}\right)_{j \geq 1}\right\|_{l^{2}} \\
& \leq C\left(\|\nabla \phi\|_{L^{\infty}\left(\mathbb{R}^{n}\right)}\|\nabla \psi\|_{H^{s-1}\left(\mathbb{R}^{n}\right)}+\|\nabla \phi\|_{H^{s-1}\left(\mathbb{R}^{n}\right)}\|\nabla \psi\|_{L^{\infty}\left(\mathbb{R}^{n}\right)}\right) .
\end{aligned}
$$

(3) [4, Corollary 2.86] For any $s>0$, the following product estimate holds true

$$
\|\phi \psi\|_{H^{s}\left(\mathbb{R}^{n}\right)} \leq C\left(\|\phi\|_{L^{\infty}\left(\mathbb{R}^{n}\right)}\|\psi\|_{H^{s}\left(\mathbb{R}^{n}\right)}+\|\phi\|_{H^{s}\left(\mathbb{R}^{n}\right)}\|\psi\|_{L^{\infty}\left(\mathbb{R}^{n}\right)}\right) .
$$

(4) $\left[4\right.$, Theorems 2.87 and Theorem 2.89] For any $s>0$ and $g \in C^{k+1}$ with $k=[s] \in \mathbb{N}$, the following composition estimate holds true

$$
\|\nabla(g \circ \theta)\|_{H^{s-1}\left(\mathbb{R}^{n}\right)} \leq C\left(g,\|\theta\|_{L^{\infty}\left(\mathbb{R}^{n}\right)}\right)\|\nabla \theta\|_{H^{s-1}\left(\mathbb{R}^{n}\right)} .
$$

If $g \in C_{b}^{k+1}$ with $k=[s] \in \mathbb{N}$, then the above estimate can be improved in the spatial dimension two as follows

$$
\|\nabla(g \circ \theta)\|_{H^{s-1}\left(\mathbb{R}^{2}\right)} \leq C\left(\|g\|_{C^{k+1}},\|\theta\|_{H^{1}\left(\mathbb{R}^{2}\right)}\right)\|\nabla \theta\|_{H^{s-1}\left(\mathbb{R}^{2}\right)} .
$$

The commutator estimate (2.28) will present its power in the low regularity regime (see Sects. 2.3.12.3.3 below), and the classical commutator estimate (2.29) will help in the high regularity regime (see Sect. 2.3.4 below).

The composition estimate (2.32) will help to bound the diffusion coefficients $\kappa, \mu$ in terms of $\theta$ in the low regularity regime, where only $H^{1}\left(\mathbb{R}^{2}\right)$-norm (instead of $L_{x}^{\infty}$-norm) of $\theta$ is available.

2.3.1. Estimates for the general parabolic equations. We derive in this paragraph a priori $H^{s}, s \in(0,2)$ Estimates for a general linear parabolic equation, which should be of independent interest.

Lemma 2.2. Let $\psi=\psi(t, x):[0, \infty) \times \mathbb{R}^{2} \mapsto \mathbb{R}^{m}, m \geq 1$ be a smooth solution with sufficiently decay of the following linear parabolic equation

$$
\left\{\begin{array}{l}
\partial_{t} \psi+u \cdot \nabla_{x} \psi-\operatorname{div}_{x}\left(\kappa \nabla_{x} \psi\right)=f \\
\left.\psi\right|_{t=0}=\psi_{0}
\end{array}\right.
$$

where

- $u=u(t, x): \mathbb{R}^{+} \times \mathbb{R}^{2} \mapsto \mathbb{R}^{2}$ is a given divergence-free vector field: div $u=0$;

- $\kappa=\kappa(t, x): \mathbb{R}^{+} \times \mathbb{R}^{2} \rightarrow\left[\kappa_{*}, \kappa^{*}\right]$ with $\kappa_{*}, \kappa^{*} \in(0, \infty)$;

- $f=f(t, x): \mathbb{R}^{+} \times \mathbb{R}^{2} \mapsto \mathbb{R}^{m}$ denotes the given external force.

Then, the following a priori $H_{x}^{s}$-Estimates for (2.33) holds true:

$$
\begin{gathered}
\|\psi\|_{L_{T}^{\infty} H_{x}^{s}}^{2}+\|\nabla \psi\|_{L_{T}^{2} H_{x}^{s}}^{2} \leq C\left(\kappa_{*}\right)\left(\left\|\psi_{0}\right\|_{H_{x}^{s}}^{2}+\|f\|_{L_{T}^{2} H_{x}^{s-1}}^{2}\right) \\
\times \exp \left(C\left(\kappa_{*}, s, \nu\right)\left(\|\nabla u\|_{L_{T}^{2} L_{x}^{2}}^{2}+\|\nabla \kappa\|_{L_{T}^{2 / \nu} H_{x}^{\nu}}^{2 / 2}+\|f\|_{L_{T}^{1} H_{x}^{-s}}\right)\right) \\
\quad \text { for any } s \in(0,2) \text { and } \nu \in(s-1,1) \subset(-1,1) .
\end{gathered}
$$

Proof. It is straightforward to derive the following $L_{x}^{2}$-Estimate by simply taking the $L^{2}\left(\mathbb{R}^{2}\right)$ inner product of the equation (2.33) and $\psi$ itself

$$
\|\psi\|_{L_{T}^{\infty} L_{x}^{2}}^{2}+\|\nabla \psi\|_{L_{T}^{2} L_{x}^{2}}^{2} \leq C\left(\kappa_{*}\right)\left(\left\|\psi_{0}\right\|_{L_{x}^{2}}^{2}+\int_{0}^{T}\langle\psi, f\rangle_{H_{x}^{s}, H_{x}^{-s}} \mathrm{~d} t\right), \quad \forall s \in \mathbb{R} .
$$

We next consider the a priori estimates for the $H^{s}\left(\mathbb{R}^{2}\right)$-norm. By virtue of the description (2.26) of the $H^{s}\left(\mathbb{R}^{2}\right)$-norm, we consider the dyadic piece of $\psi$ :

$$
\psi_{j}:=\Delta_{j} \psi, \quad j \geq 0
$$


where the operator $\Delta_{j}$ is defined in (2.24). We apply $\Delta_{j}$ to the linear $\psi$-equation to derive the equation for $\psi_{j}$ :

$$
\partial_{t} \psi_{j}+u \cdot \nabla \psi_{j}-\operatorname{div}\left(\kappa \nabla \psi_{j}\right)=\left[u, \Delta_{j}\right] \cdot \nabla \psi-\operatorname{div}\left(\left[\kappa, \Delta_{j}\right] \nabla \psi\right)+f_{j}, \quad j \geq 0 .
$$

We take the $L^{2}$ inner product of the equation (2.37) and $\psi_{j}$ and make use of $\operatorname{div} u=0$ and $\kappa \geq \kappa_{*}$ to derive

$$
\begin{aligned}
& \frac{1}{2} \frac{\mathrm{d}}{\mathrm{d} t}\left\|\psi_{j}\right\|_{L_{x}^{2}}^{2}+\kappa_{*}\left\|\nabla \psi_{j}\right\|_{L_{x}^{2}}^{2} \leq\left\|\psi_{j}\right\|_{L_{x}^{2}}\left\|\left[u, \Delta_{j}\right] \cdot \nabla \psi\right\|_{L_{x}^{2}} \\
& \quad+\left\|\nabla \psi_{j}\right\|_{L_{x}^{2}}\left\|\left[\kappa, \Delta_{j}\right] \nabla \psi\right\|_{L_{x}^{2}}+\left\|f_{j}\right\|_{L_{x}^{2}}\left\|\psi_{j}\right\|_{L_{x}^{2}}, j \geq 0 .
\end{aligned}
$$

By use of Bernstein's inequality (2.25), we have

$$
\begin{aligned}
& \frac{\mathrm{d}}{\mathrm{d} t}\left\|\psi_{j}\right\|_{L_{x}^{2}}^{2}+2^{2 j}\left\|\psi_{j}\right\|_{L_{x}^{2}}^{2} \\
& \quad \leq C\left(\kappa_{*}\right)\left\|\psi_{j}\right\|_{L_{x}^{2}}\left(\left\|\left[u, \Delta_{j}\right] \cdot \nabla \psi\right\|_{L_{x}^{2}}+2^{j}\left\|\left[\kappa, \Delta_{j}\right] \nabla \psi\right\|_{L_{x}^{2}}+\left\|f_{j}\right\|_{L_{x}^{2}}\right),
\end{aligned}
$$

that is,

$$
\begin{aligned}
& \frac{\mathrm{d}}{\mathrm{d} t}\left\|\psi_{j}\right\|_{L_{x}^{2}}+2^{2 j}\left\|\psi_{j}\right\|_{L_{x}^{2}} \\
& \quad \leq C\left(\kappa_{*}\right)\left(\left\|\left[u, \Delta_{j}\right] \cdot \nabla \psi\right\|_{L_{x}^{2}}+2^{j}\left\|\left[\kappa, \Delta_{j}\right] \nabla \psi\right\|_{L_{x}^{2}}+\left\|f_{j}\right\|_{L_{x}^{2}}\right), \quad j \geq 0 .
\end{aligned}
$$

We make use of the commutator estimate (2.28) in Lemma 2.1 to estimate the commutators $\|\left[u, \Delta_{j}\right]$. $\nabla \psi \|_{L_{x}^{2}}$ and $2^{j}\left\|\left[\kappa, \Delta_{j}\right] \nabla \psi_{j}\right\|_{L_{x}^{2}}$ in the above inequality in the following way. Let $\left(l_{j}\right)_{j \geq 0}$ be a normalized sequence in $\ell^{1}(\mathbb{N})$ such that $l_{j} \geq 0$ and $\sum_{j \geq 0} l_{j}=1$. Then, we have

$$
\begin{aligned}
& \left\|\left[u, \Delta_{j}\right] \nabla \psi\right\|_{L^{2}} \leq C(s) l_{j} 2^{j(1-s)}\|\nabla u\|_{L_{x}^{2}}\|\nabla \psi\|_{H_{x}^{s-1}}, \text { for } s \in(0,2), \\
& 2^{j}\left\|\left[\kappa, \Delta_{j}\right] \nabla \psi\right\|_{L_{x}^{2}} \leq C(s, \nu) l_{j} 2^{j(1-s)}\|\nabla \kappa\|_{H_{x}^{\nu}}\|\nabla \psi\|_{H_{x}^{s-\nu}} \\
& \quad \text { for } \nu \in(-1,1), s \in(-1, \nu+1) .
\end{aligned}
$$

Therefore, we have

$$
\begin{aligned}
& \frac{\mathrm{d}}{\mathrm{d} t}\left\|\psi_{j}\right\|_{L_{x}^{2}}+2^{2 j}\left\|\psi_{j}\right\|_{L_{x}^{2}} \\
& \quad \leq C\left(\kappa_{*}, s, \nu\right) l_{j} 2^{j(1-s)}\left(\|\nabla u\|_{L_{x}^{2}}\|\nabla \psi\|_{H_{x}^{s-1}}+\|\nabla \kappa\|_{H_{x}^{\nu}}\|\nabla \psi\|_{H_{x}^{s-\nu}}\right)+C\left(\kappa_{*}\right)\left\|f_{j}\right\|_{L_{x}^{2}} \\
& \quad \text { for } \nu \in(-1,1), s \in(0, \nu+1), j \geq 0 .
\end{aligned}
$$

We use Duhamel's Principle to derive

$$
\begin{aligned}
& \left\|\psi_{j}\right\|_{L_{x}^{2}} \leq e^{-t 2^{2 j}}\left\|\left(\psi_{0}\right)_{j}\right\|_{L_{x}^{2}}+C\left(\kappa_{*}\right) \int_{0}^{t} e^{-(t-\tau) 2^{2 j}}\left\|f_{j}(\tau)\right\|_{L_{x}^{2}} d \tau \\
& +C\left(\kappa_{*}, s, \nu\right) 2^{j(1-s)} l_{j} \int_{0}^{t} e^{-(t-\tau) 2^{2 j}}\left(\|\nabla u(\tau)\|_{L_{x}^{2}}\|\nabla \psi(\tau)\|_{H_{x}^{s-1}}\right. \\
& \left.+\|\nabla \kappa(\tau)\|_{H_{x}^{\nu}}\|\nabla \psi(\tau)\|_{H_{x}^{s-\nu}}\right) d \tau, \quad j \geq 0 .
\end{aligned}
$$


We multiply the inequality $(2.40)$ by $2^{j s}$ to derive

$$
\begin{aligned}
& 2^{j s}\left\|\psi_{j}\right\|_{L_{x}^{2}} \leq 2^{j s} e^{-t 2^{2 j}}\left\|\left(\psi_{0}\right)_{j}\right\|_{L_{x}^{2}}+C\left(\kappa_{*}\right) 2^{j s} \int_{0}^{t} e^{-(t-\tau) 2^{2 j}}\left\|f_{j}\right\|_{L_{x}^{2}} d \tau \\
& \quad+C\left(\kappa_{*}, s, \nu\right) 2^{j} l_{j} \int_{0}^{t} e^{-(t-\tau) 2^{2 j}}\left(\|\nabla u(\tau)\|_{L_{x}^{2}}\|\nabla \psi(\tau)\|_{H_{x}^{s-1}}\right. \\
& \left.\quad+\|\nabla \kappa(\tau)\|_{H_{x}^{\nu}}\|\nabla \psi(\tau)\|_{H_{x}^{s-\nu}}\right) d \tau, \quad j \geq 0 .
\end{aligned}
$$

We take $L^{\infty}([0, T])$-norm in $t$ of $(2.41)$ and the $L^{2}([0, T])$-norm in $t$ of $2^{j} \cdot(2.41)$, to derive by use of Young's inequality that

$$
\begin{aligned}
& 2^{j s}\left\|\psi_{j}\right\|_{L_{T}^{\infty} L_{x}^{2}}+2^{j(s+1)}\left\|\psi_{j}\right\|_{L_{T}^{2} L_{x}^{2}} \leq 2^{j s}\left\|\left(\psi_{0}\right)_{j}\right\|_{L_{x}^{2}}+C\left(\kappa_{*}\right) 2^{j(s-1)}\left\|f_{j}\right\|_{L_{T}^{2} L_{x}^{2}} \\
& \quad+C\left(\kappa_{*}, s, \nu\right) l_{j}\|\| \nabla u\left\|_{L_{x}^{2}}\right\| \nabla \psi\left\|_{H_{x}^{s-1}}+\right\| \nabla \kappa\left\|_{H_{x}^{\nu}}\right\| \nabla \psi\left\|_{H_{x}^{s-\nu}}\right\|_{L_{T}^{2}} .
\end{aligned}
$$

We take square of (2.42) and sum them up for $j \in \mathbb{N}$ to derive

$$
\begin{aligned}
& \sum_{j \geq 0}\left(2^{2 j s}\left\|\psi_{j}\right\|_{L_{T}^{\infty} L_{x}^{2}}^{2}+2^{2 j(s+1)}\left\|\psi_{j}\right\|_{L_{T}^{2} L_{x}^{2}}^{2}\right) \\
& \lesssim_{\kappa_{*}, s, \nu} \sum_{j \geq 0}\left(2^{2 j s}\left\|\left(\psi_{0}\right)_{j}\right\|_{L_{x}^{2}}^{2}+2^{2 j(s-1)}\left\|f_{j}\right\|_{L_{T}^{2} L_{x}^{2}}^{2}\right) \\
& \quad+\int_{0}^{T}\|\nabla u\|_{L_{x}^{2}}^{2}\|\nabla \psi\|_{H_{x}^{s-1}}^{2}+\|\nabla \kappa\|_{H_{x}^{\nu}}^{2}\|\nabla \psi\|_{H_{x}^{s-\nu}}^{2} \mathrm{~d} t, \quad j \geq 0
\end{aligned}
$$

that is, by virtue of the $L^{2}$-Estimate $(2.35)$,

$$
\begin{aligned}
& \|\psi\|_{L_{T}^{\infty} H_{x}^{s}}^{2}+\|\nabla \psi\|_{L_{T}^{2} H_{x}^{s}}^{2} \lesssim_{\kappa_{*}, s, \nu}\left(\left\|\psi_{0}\right\|_{H_{x}^{s}}^{2}+\|f\|_{L_{T}^{2} H_{x}^{s-1}}^{2}+\int_{0}^{T}\|\psi\|_{H_{x}^{s}}\|f\|_{H_{x}^{-s}} \mathrm{~d} t\right. \\
& \left.+\int_{0}^{T}\|\nabla u\|_{L_{x}^{2}}^{2}\|\nabla \psi\|_{H_{x}^{s-1}}^{2}+\|\nabla \kappa\|_{H_{x}^{\nu}}^{2}\|\nabla \psi\|_{H_{x}^{s-\nu}}^{2} \mathrm{~d} t\right) .
\end{aligned}
$$

We next consider the norm $\|\nabla \psi\|_{H_{x}^{s-\nu}}$. By the interpolation inequality (2.27), we have

$$
\|\nabla \psi\|_{H_{x}^{s-\nu}} \leq C\|\nabla \psi\|_{H_{x}^{s-1}}^{\nu}\|\nabla \psi\|_{H_{x}^{s}}^{1-\nu}, \quad \nu \in(0,1),
$$

which implies by Young's inequality that

$$
\begin{aligned}
\int_{0}^{T}\|\nabla \kappa\|_{H_{x}^{\nu}}^{2}\|\nabla \psi\|_{H_{x}^{s-\nu}}^{2} \mathrm{~d} t & \leq \int_{0}^{T}\|\nabla \kappa\|_{H_{x}^{\nu}}^{2}\|\nabla \psi\|_{H_{x}^{s-1}}^{2 \nu}\|\nabla \psi\|_{H_{x}^{s}}^{2(1-\nu)} \mathrm{d} t \\
& \leq \varepsilon\|\nabla \psi\|_{L_{T}^{2} H_{x}^{s}}^{2}+C_{\varepsilon} \int_{0}^{T}\|\nabla \kappa\|_{H_{x}^{\nu}}^{2 / \nu}\|\nabla \psi\|_{H_{x}^{s-1}}^{2} \mathrm{~d} t .
\end{aligned}
$$

To conclude, by taking $\varepsilon$ small enough and Gronwall's inequality, we derive the $H^{s}$-Estimate (2.34). 
2.3.2. Case $\left(\theta_{0}, u_{0}\right) \in H^{s}\left(\mathbb{R}^{2}\right) \times\left(L^{2}\left(\mathbb{R}^{2}\right)\right)^{2}, s \in(1,2]$. In this subsection, we are going to prove the $H^{s}$-Estimates $(1.17)$ for the unique solution $(\theta, u)$ of the Boussinesq equations (1.1) with the initial data $\left(\theta_{0}, u_{0}\right) \in H^{s}\left(\mathbb{R}^{2}\right) \times\left(L^{2}\left(\mathbb{R}^{2}\right)\right)^{2}, s \in(1,2)$, following exactly the procedure in Sect. 2.3.1. We will pay more attention on the "nonlinearities" in the equations such as $\kappa=a(\theta), u \cdot \nabla u$ when using the commutator estimates and will sketch the proof.

The endpoint estimate (1.18) for $\left(\theta_{0}, u_{0}\right) \in H^{2}\left(\mathbb{R}^{2}\right) \times\left(L^{2}\left(\mathbb{R}^{2}\right)\right)^{2}$ follows similarly as in the proof for the $H^{1}$-Estimate for $\theta$ in Sect. 2.2 and we will sketch the proof.

Case $\left(\theta_{0}, u_{0}\right) \in H^{s}\left(\mathbb{R}^{2}\right) \times\left(L^{2}\left(\mathbb{R}^{2}\right)\right)^{2}, s \in(1,2)$

Similarly as (2.38), we have the following preliminary estimate for $\theta_{j}=\Delta_{j} \theta$ :

$$
\frac{\mathrm{d}}{\mathrm{d} t}\left\|\theta_{j}\right\|_{L_{x}^{2}}+2^{2 j}\left\|\theta_{j}\right\|_{L_{x}^{2}} \leq C\left(\kappa_{*}\right)\left(\left\|\left[u, \Delta_{j}\right] \cdot \nabla \theta\right\|_{L_{x}^{2}}+2^{j}\left\|\left[\kappa, \Delta_{j}\right] \nabla \theta\right\|_{L_{x}^{2}}\right), \quad j \geq 0 .
$$

By use of the commutator estimates (2.39) and the action estimate (2.32):

$$
\|\nabla \kappa\|_{H^{\nu}} \leq C\left(\|a\|_{C^{2}},\|\theta\|_{H^{1}}\right)\|\nabla \theta\|_{H^{\nu}} \text { for } \nu \in(0,1),
$$

we derive similar as $(2.42)$

$$
\begin{aligned}
& 2^{2 j s}\left\|\theta_{j}\right\|_{L_{T}^{\infty} L_{x}^{2}}^{2}+2^{2 j(s+1)}\left\|\theta_{j}\right\|_{L_{T}^{2} L_{x}^{2}}^{2} \leq 2^{2 j s}\left\|\left(\theta_{0}\right)_{j}\right\|_{L_{x}^{2}}^{2} \\
& +C\left(\kappa_{*}, s, \nu,\|a\|_{C^{2}},\|\theta\|_{L_{T}^{\infty} H_{x}^{1}}\right)\left(l_{j}\right)^{2} \int_{0}^{T}\left(\|\nabla u\|_{L_{x}^{2}}^{2}\|\nabla \theta\|_{H_{x}^{s-1}}^{2}\right. \\
& \left.+\|\nabla \theta\|_{H_{x}^{\nu}}^{2}\|\nabla \theta\|_{H_{x}^{s-\nu}}^{2}\right) \mathrm{d} t, \quad 1<s<\nu+1<2 .
\end{aligned}
$$

By using the interpolation inequality (2.27), we have

$$
\|\nabla \theta\|_{H_{x}^{\nu}}\|\nabla \theta\|_{H_{x}^{s-\nu}} \leq C\|\nabla \theta\|_{L_{x}^{2}}^{1-\nu}\|\nabla \theta\|_{H_{x}^{1}}^{\nu}\|\nabla \theta\|_{H_{x}^{s-1}}^{\nu}\|\nabla \theta\|_{H_{x}^{s}}^{1-\nu}, \quad 0<\nu<1 .
$$

Recall the $L^{2}$-Estimate (2.16) for $\theta$ :

$$
\|\theta\|_{L_{T}^{\infty} L_{x}^{2}}^{2}+\|\nabla \theta\|_{L_{T}^{2} L_{x}^{2}}^{2} \leq C\left(\kappa_{*}\right)\left\|\theta_{0}\right\|_{L_{x}^{2}}^{2} .
$$

Therefore by Young's inequality, we arrive at

$$
\begin{aligned}
& \|\theta\|_{L_{T}^{\infty} H_{x}^{s}}^{2}+\|\nabla \theta\|_{L_{T}^{2} H_{x}^{s}}^{2} \leq C\left(\kappa_{*}\right)\left\|\theta_{0}\right\|_{H_{x}^{s}}^{2} \\
& +C\left(\kappa_{*}, s, \nu,\|a\|_{C^{2}},\|\theta\|_{L_{T}^{\infty} H_{x}^{1}}\right) \int_{0}^{T}\left(\|\nabla u\|_{L_{x}^{2}}^{2}+\|\nabla \theta\|_{H_{x}^{1}}^{2}\right)\|\nabla \theta\|_{H_{x}^{s-1}}^{2} \mathrm{~d} t,
\end{aligned}
$$

which, together with Gronwall's inequality, implies (1.17).

Endpoint case $\left(\theta_{0}, u_{0}\right) \in H^{2}\left(\mathbb{R}^{2}\right) \times\left(L^{2}\left(\mathbb{R}^{2}\right)\right)^{2}$

We recall the function $\eta=A^{-1}(\theta)$ defined in (2.11), and the parabolic $\eta$-equation (2.15):

$$
\partial_{t} \eta+u \cdot \nabla \eta-\kappa \Delta \eta=0 .
$$

We are going to derive the a priori $H^{2}$-Estimate for $\eta$ under the conditions

$$
\operatorname{div} u=0, \quad \nabla u \in L_{\mathrm{loc}}^{2}\left([0, \infty) ;\left(L^{2}\left(\mathbb{R}^{2}\right)\right)^{4}\right) \text { and } \nabla \kappa \in L_{\mathrm{loc}}^{4}\left([0, \infty) ;\left(L^{4}\left(\mathbb{R}^{2}\right)\right)^{2}\right) .
$$

We test the above $\eta$-equation $(2.46)$ by $\Delta^{2} \eta$, to arrive at

$$
\frac{1}{2} \frac{\mathrm{d}}{\mathrm{d} t} \int_{\mathbb{R}^{2}}|\Delta \eta|^{2} \mathrm{~d} x+\int_{\mathbb{R}^{2}} \kappa|\nabla \Delta \eta|^{2} \mathrm{~d} x=-\int_{\mathbb{R}^{2}}\left(u \cdot \nabla \eta \Delta^{2} \eta+\nabla \kappa \cdot \nabla \Delta \eta \Delta \eta\right) \mathrm{d} x .
$$


By integration by parts, $\operatorname{div} u=0$ and the embedding $L^{1}\left(\mathbb{R}^{2}\right) \hookrightarrow H^{-1}\left(\mathbb{R}^{2}\right)$, we derive

$$
\begin{aligned}
-\int_{\mathbb{R}^{2}} u \cdot \nabla \eta \Delta^{2} \eta \mathrm{d} x & =\int_{\mathbb{R}^{2}} \nabla \Delta \eta \cdot \nabla u \cdot \nabla \eta-\nabla u: \nabla^{2} \eta \Delta \eta \mathrm{d} x \\
& \leq 4\|\nabla u\|_{L_{x}^{2}}\left(\|\nabla \Delta \eta\|_{L_{x}^{2}}\|\nabla \eta\|_{H_{x}^{1}}+\left\|\nabla^{2} \eta\right\|_{L_{x}^{4}}^{2}\right) \\
& \leq \frac{\kappa_{*}}{4}\|\nabla \Delta \eta\|_{L_{x}^{2}}^{2}+C\left(\kappa_{*}\right)\|\nabla u\|_{L_{x}^{2}}^{2}\|\nabla \eta\|_{H_{x}^{1}}^{2} .
\end{aligned}
$$

Similarly, we have

$$
\begin{aligned}
-\int_{\mathbb{R}^{2}} \nabla \kappa \cdot \nabla \Delta \eta \Delta \eta \mathrm{d} x & \leq\|\nabla \kappa\|_{L_{x}^{4}}\|\nabla \Delta \eta\|_{L_{x}^{2}}\|\Delta \eta\|_{L_{x}^{4}} \\
& \leq \frac{\kappa_{*}}{4}\|\nabla \Delta \eta\|_{L_{x}^{2}}^{2}+C\left(\kappa_{*}\right)\|\nabla \kappa\|_{L_{x}^{4}}^{4}\|\Delta \eta\|_{L_{x}^{2}}^{2} .
\end{aligned}
$$

To conclude, we have the following a priori $\dot{H}_{x}^{2}$-Estimate for $\eta$ and any positive time $T>0$ by Gronwall's inequality

$$
\begin{aligned}
\|\Delta \eta(T)\|_{L_{x}^{2}}^{2}+\|\nabla \Delta \eta\|_{L_{T}^{2} L_{x}^{2}}^{2} \leq & C\left(\kappa_{*}\right)\left(\left\|\Delta \eta_{0}\right\|_{L_{x}^{2}}^{2}+\|\nabla u\|_{L_{T}^{2} L_{x}^{2}}^{2}\|\nabla \eta\|_{L_{T}^{\infty} L_{x}^{2}}^{2}\right) \\
& \times \exp \left(C\left(\kappa_{*}\right)\left(\|\nabla u\|_{L_{T}^{2} L_{x}^{2}}^{2}+\|\nabla \kappa\|_{L_{T}^{4} L_{x}^{4}}^{4}\right)\right) .
\end{aligned}
$$

By view of the equivalence relation $(2.12)$ as well $\mathrm{as}^{5}$

$$
\left\|\nabla^{3} \theta\right\|_{L_{T}^{2} L_{x}^{2}} \leq C\left(\kappa_{*},\|a\|_{C^{2}}\right)\left(\left(\|\nabla \eta\|_{L_{T}^{4} L_{x}^{4}}^{2}+\left\|\nabla^{2} \eta\right\|_{L_{T}^{2} L_{x}^{2}}\right)\|\nabla \eta\|_{L_{T}^{\infty} L_{x}^{2}}+\left\|\nabla^{3} \eta\right\|_{L_{T}^{2} L_{x}^{2}}\right),
$$

we derive the $H^{2}$-Estimate (1.18) for $\theta$ by virtue of the $H^{1}$-Estimate (2.18):

$$
\begin{aligned}
& \|\theta\|_{L_{T}^{\infty} H_{x}^{2}}^{2}+\|\nabla \theta\|_{L_{T}^{2} H_{x}^{2}}^{2} \leq C\left(\kappa_{*},\|a\|_{C^{2}}, \kappa^{*}\right)\left\|\theta_{0}\right\|_{H^{2}}^{2}\left(1+\left\|\nabla \theta_{0}\right\|_{L^{2}}^{2}\right)^{2} \\
& \quad \times \exp \left(C\left(\kappa_{*},\|a\|_{C^{1}}\right)\left(\|\nabla u\|_{L_{T}^{2} L_{x}^{2}}^{2}+\|u\|_{L_{T}^{4} L_{x}^{4}}^{4}+\|\nabla \theta\|_{L_{T}^{4} L_{x}^{4}}^{4}\right)\right) .
\end{aligned}
$$

2.3.3. Case $\left(\boldsymbol{\theta}_{0}, \boldsymbol{u}_{0}\right) \in \boldsymbol{H}^{1}\left(\mathbb{R}^{2}\right) \times\left(\boldsymbol{H}^{s}\left(\mathbb{R}^{2}\right)\right)^{2}, s \in(\mathbf{0}, 2]$. In this subsection we are going to sketch the proof of the $H^{s}, s \in(0,2)$-Estimate (1.19) for the divergence-free vector field $u$ of the unique solution $(\theta, u)$ to the Boussinesq equations (1.1), under the assumption that $\theta_{0} \in H^{1}\left(\mathbb{R}^{2}\right)$, following the procedure in Sect. 2.3.1.

We deal with the endpoint case $\left(\theta_{0}, u_{0}\right) \in H^{1}\left(\mathbb{R}^{2}\right) \times\left(H^{2}\left(\mathbb{R}^{2}\right)\right)^{2}$ similarly as for the endpoint case above.

Case $\left(\theta_{0}, u_{0}\right) \in H^{1}\left(\mathbb{R}^{2}\right) \times\left(H^{s}\left(\mathbb{R}^{2}\right)\right)^{2}, s \in(0,2)$

Recall (2.5) for the definition of the Leray-Helmholtz projector $\mathbb{P}$ such that

$$
\mathbb{P} u=u, \quad \mathbb{P} \nabla \Pi=0 .
$$

We apply $\mathbb{P}$ to the velocity equation $(1.1)_{2}$ to arrive at

$$
\partial_{t} u+\mathbb{P}(u \cdot \nabla u)-\mathbb{P} \operatorname{div}(\mu S u)=\mathbb{P}\left(\theta \overrightarrow{e_{2}}\right) .
$$

\footnotetext{
${ }^{5}$ It is also straightforward to calculate

$$
\begin{aligned}
\partial_{j k l} \eta= & a^{\prime \prime}(\theta)\left(\partial_{j} \theta \partial_{k} \theta \partial_{l} \theta\right)+a^{\prime}(\theta)\left(\partial_{j k} \theta \partial_{l} \theta+\partial_{j l} \partial_{k} \theta+\partial_{k l} \theta \partial_{j} \theta\right)+a(\theta) \partial_{j k l} \theta \\
\partial_{j k l} \theta= & \left(-\frac{a^{\prime \prime}\left(A^{-1}(\eta)\right)}{a^{4}\left(A^{-1}(\eta)\right)}+\frac{3\left(a^{\prime}\left(A^{-1}(\eta)\right)\right)^{2}}{a^{5}\left(A^{-1}(\eta)\right)}\right) \partial_{j} \eta \partial_{k} \eta \partial_{l} \eta \\
& -\frac{a^{\prime}\left(A^{-1}(\eta)\right)}{a^{3}\left(A^{-1}(\eta)\right)}\left(\partial_{j k} \eta \partial_{l} \eta+\partial_{j l} \eta \partial_{l} \eta+\partial_{k l} \eta \partial_{j} \eta\right)+\frac{1}{a\left(A^{-1}(\eta)\right)} \partial_{j k l} \eta .
\end{aligned}
$$
}


We apply $\Delta_{j}$ to the above equation (2.47) to arrive at the equation for $u_{j}:=\Delta_{j} u$

$$
\partial_{t} u_{j}+\mathbb{P} u \cdot \nabla u_{j}-\mathbb{P} \operatorname{div}\left(\mu S u_{j}\right)=\mathbb{P}\left[u, \Delta_{j}\right] \cdot \nabla u-\mathbb{P} \operatorname{div}\left(\left[\mu, \Delta_{j}\right] S u\right)+\mathbb{P}\left(\theta_{j} \overrightarrow{e_{2}}\right) .
$$

We take the $L^{2}\left(\mathbb{R}^{2}\right)$-inner product between $(2.48)$ and the divergence-free dyadic piece $u_{j}=\mathbb{P} u_{j}$ and follow the similar argument as to arrive at (2.38), to deduce

$$
\frac{\mathrm{d}}{\mathrm{d} t}\left\|u_{j}\right\|_{L_{x}^{2}}+2^{2 j}\left\|u_{j}\right\|_{L_{x}^{2}} \leq C\left(\mu_{*}\right)\left(\left\|\left[u, \Delta_{j}\right] \cdot \nabla u\right\|_{L_{x}^{2}}+2^{j}\left\|\left[\mu, \Delta_{j}\right] \nabla u\right\|_{L_{x}^{2}}+\left\|\theta_{j}\right\|_{L_{x}^{2}}\right) .
$$

By use of the commutator estimate (2.28) in Lemma 2.1 again, we have the following commutator estimates as in (2.39):

$$
\begin{aligned}
& \left\|\left[u, \Delta_{j}\right] \nabla u\right\|_{L_{x}^{2}} \leq C l_{j} 2^{j(1-s)}\|\nabla u\|_{L_{x}^{2}}\|\nabla u\|_{H_{x}^{s-1}}, \text { for } s \in(0,2), \\
& 2^{j}\left\|\left[\mu, \Delta_{j}\right] \nabla u\right\|_{L_{x}^{2}} \leq C l_{j} 2^{j(1-s)}\|\nabla \mu\|_{H_{x}^{\nu}}\|\nabla u\|_{H_{x}^{s-\nu}}, \text { for } \nu \in(-1,1), s \in(-1, \nu+1) .
\end{aligned}
$$

By virtue of the composition estimate (2.32) in Lemma 2.1:

$$
\|\nabla \mu\|_{H_{x}^{\nu}} \leq C\left(\|b\|_{C^{[\nu]+2}},\|\theta\|_{H^{1}}\right)\|\nabla \theta\|_{H_{x}^{\nu}}
$$

we derive similar as (2.42) that, for $0<s<\nu+1<2$,

$$
\begin{aligned}
& 2^{2 j s}\left\|u_{j}\right\|_{L_{T}^{\infty} L_{x}^{2}}^{2}+2^{2 j(s+1)}\left\|u_{j}\right\|_{L_{T}^{2} L_{x}^{2}}^{2} \leq 2^{2 j s}\left\|\left(u_{0}\right)_{j}\right\|_{L_{x}^{2}}^{2} \\
& \quad+C\left(\mu_{*}\right) \int_{0}^{T} 2^{2 j(s-1)}\left\|\theta_{j}\right\|_{L_{x}^{2}}^{2} \mathrm{~d} t+C\left(\mu_{*}, s, \nu,\|b\|_{\left.C^{[\nu]+2},\|\theta\|_{L_{T}^{\infty} H_{x}^{1}}\right)\left(l_{j}\right)^{2}}\right. \\
& \quad \times \int_{0}^{T}\left(\|\nabla u\|_{L_{x}^{2}}^{2}\|\nabla u\|_{H_{x}^{s-1}}^{2}+\|\nabla \theta\|_{H_{x}^{\nu}}^{2}\|\nabla u\|_{H_{x}^{s-\nu}}^{2}\right) \mathrm{d} t .
\end{aligned}
$$

By the interpolation inequality $(2.27)$ :

$$
\|\nabla \theta\|_{H_{x}^{\nu}}\|\nabla u\|_{H_{x}^{s-\nu}} \leq C\|\nabla \theta\|_{L_{x}^{2}}^{1-\nu}\|\nabla \theta\|_{H_{x}^{1}}^{\nu}\|\nabla u\|_{H_{x}^{s-1}}^{\nu}\|\nabla u\|_{H_{x}^{s}}^{1-\nu}, \quad \text { for } \nu \in(0,1),
$$

and the $L^{2}$-Estimate (1.13):

$$
\|u\|_{L_{T}^{\infty} L_{x}^{2}}^{2}+\|\nabla u\|_{L_{T}^{2} L_{x}^{2}}^{2} \leq C\left(\mu_{*}\right)\left(\left\|u_{0}\right\|_{L_{x}^{2}}^{2}+T\left\|\theta_{0}\right\|_{L_{x}^{2}}^{2}\right),
$$

we arrive at the following by Young's inequality

$$
\begin{gathered}
\|u\|_{L_{T}^{\infty} H_{x}^{s}}^{2}+\|\nabla u\|_{L_{T}^{2} H_{x}^{s}}^{2} \leq C\left(\mu_{*}\right)\left(\left\|u_{0}\right\|_{H_{x}^{s}}^{2}+T\left\|\theta_{0}\right\|_{L_{x}^{2}}^{2}+\|\theta\|_{L_{T}^{2} H_{x}^{s-1}}^{2}\right) \\
\quad+C\left(\mu_{*}, s, \nu,\|b\|_{C^{2}},\|\theta\|_{L_{T}^{\infty} H_{x}^{1}}\right) \int_{0}^{T}\left(\|\nabla u\|_{L_{x}^{2}}^{2}+\|\nabla \theta\|_{H_{x}^{1}}^{2}\right)\|\nabla u\|_{H_{x}^{s-1}}^{2} \mathrm{~d} t
\end{gathered}
$$

which, together with Gronwall's inequality, implies (1.19).

Endpoint case $\left(\theta_{0}, u_{0}\right) \in H^{1}\left(\mathbb{R}^{2}\right) \times\left(H^{2}\left(\mathbb{R}^{2}\right)\right)^{2}$

We recall the $u$-equation (2.47) where

$$
\operatorname{div}(\mu S u)=\mu \Delta u+\nabla \mu \cdot S u .
$$

We test (2.47) by the divergence-free vector field $\Delta^{2} u$, to arrive at

$$
\begin{aligned}
& \frac{1}{2} \frac{\mathrm{d}}{\mathrm{d} t} \int_{\mathbb{R}^{2}}|\Delta u|^{2} \mathrm{~d} x+\int_{\mathbb{R}^{2}} \mu|\nabla \Delta u|^{2} \mathrm{~d} x \\
& \quad=\int_{\mathbb{R}^{2}}\left(-u \cdot \nabla u \Delta^{2} u+\nabla \mu \cdot S u \cdot \Delta^{2} u-\nabla \mu \cdot \nabla \Delta u \cdot \Delta u+\Delta \theta \Delta u_{2}\right) \mathrm{d} x .
\end{aligned}
$$


By use of the embedding $L^{1}\left(\mathbb{R}^{2}\right) \hookrightarrow H^{-1}\left(\mathbb{R}^{2}\right)$ again, the right hand side can be bounded by

$$
\begin{aligned}
& C\left(\|\nabla u\|_{L_{x}^{4}}^{2}+\|u\|_{L_{x}^{4}}\left\|\nabla^{2} u\right\|_{L_{x}^{4}}+\left\|\nabla^{2} \mu\right\|_{L_{x}^{2}}\|\nabla u\|_{H_{x}^{1}}+\|\nabla \mu\|_{L_{x}^{4}}\left\|\nabla^{2} u\right\|_{L_{x}^{4}}\right)\|\nabla \Delta u\|_{L_{x}^{2}} \\
& \quad+\|\Delta \theta\|_{L_{x}^{2}}\|\Delta u\|_{L_{x}^{2}} .
\end{aligned}
$$

Thus, we have the following a priori $\dot{H}_{x}^{2}$-Estimate for $u$ and any positive time $T>0$ by Young's inequality and Gronwall's inequality

$$
\begin{aligned}
& \|\Delta u(T)\|_{L_{x}^{2}}^{2}+\|\nabla \Delta u\|_{L_{T}^{2} L_{x}^{2}}^{2} \\
& \quad \leq C\left(\mu_{*}\right)\left(\left\|\Delta u_{0}\right\|_{L_{x}^{2}}^{2}+\|\nabla u\|_{L_{T}^{4} L_{x}^{4}}^{4}+\left\|\nabla^{2} \mu\right\|_{L_{T}^{2} L_{x}^{2}}^{2}\|\nabla u\|_{L_{T}^{\infty} L_{x}^{2}}^{2}+\|\Delta \theta\|_{L_{T}^{2} L_{x}^{2}}\|\Delta u\|_{L_{T}^{2} L_{x}^{2}}\right) \\
& \quad \times \exp \left(C\left(\mu_{*}\right)\left(\|(u, \nabla \mu)\|_{L_{T}^{4} L_{x}^{4}}^{4}+\left\|\nabla^{2} \mu\right\|_{L_{T}^{2} L_{x}^{2}}^{2}\right)\right),
\end{aligned}
$$

which gives (1.20).

2.3.4. Case $\left(\boldsymbol{\theta}_{0}, u_{0}\right) \in \boldsymbol{H}^{s}\left(\mathbb{R}^{2}\right) \times\left(H^{s-2}\left(\mathbb{R}^{2}\right)\right)^{2}$ or $H^{s-1} \times\left(H^{s}\left(\mathbb{R}^{2}\right)\right)^{2}, s>2$. We are going to use the estimates in the high regularity regime in Lemma 2.1 to derive the $H^{s}$-Estimates (1.21)-(1.22)-(1.23)(1.24) in Remark 1.4. Let $\left(l_{j}^{\prime}\right)_{j \geq 0}$ be a normalized sequence in $\ell^{2}(\mathbb{N})$ such that $l_{j}^{\prime} \geq 0$ and $\sum_{j \geq 0}\left(l_{j}^{\prime}\right)^{2}=1$.

Case $\left(\theta_{0}, u_{0}\right) \in H^{s}\left(\mathbb{R}^{2}\right) \times\left(H^{s-2}\left(\mathbb{R}^{2}\right)\right)^{2}, s>2$

We can view the transport term $u \cdot \nabla \theta$ simply as a source term of the $\theta$-equation:

$$
\partial_{t} \theta-\operatorname{div}(\kappa \nabla \theta)=-u \cdot \nabla \theta
$$

Then, the preliminary estimate for $\theta_{j}=\Delta_{j} \theta$ in (2.43) can be replaced by

$$
\frac{\mathrm{d}}{\mathrm{d} t}\left\|\theta_{j}\right\|_{L_{x}^{2}}+2^{2 j}\left\|\theta_{j}\right\|_{L_{x}^{2}} \leq C\left(\kappa_{*}\right)\left(\left\|(u \cdot \nabla \theta)_{j}\right\|_{L_{x}^{2}}+2^{j}\left\|\left[\kappa, \Delta_{j}\right] \nabla \theta\right\|_{L_{x}^{2}}\right), \quad j \geq 0 .
$$

We apply Lemma 2.1 to derive the following estimates for $s>1$ :

$$
\begin{aligned}
& \left\|(u \cdot \nabla \theta)_{j}\right\|_{L_{x}^{2}} \leq C l_{j}^{\prime} 2^{j(1-s)}\left(\|u\|_{L_{x}^{\infty}}\|\nabla \theta\|_{H_{x}^{s-1}}+\|u\|_{H_{x}^{s-1}}\|\nabla \theta\|_{L_{x}^{\infty}}\right), \\
& 2^{j}\left\|\left[\kappa, \Delta_{j}\right] \nabla \theta\right\|_{L_{x}^{2}} \leq C l_{j}^{\prime} 2^{j(1-s)}\left(\|\nabla \kappa\|_{L_{x}^{\infty}}\|\nabla \theta\|_{H_{x}^{s-1}}+\|\nabla \kappa\|_{H_{x}^{s-1}}\|\nabla \theta\|_{L_{x}^{\infty}}\right) .
\end{aligned}
$$

Therefore, we have the following estimate similarly as in $(2.44)$ for $s \in(2,3)$ by virtue of $H^{s-1}\left(\mathbb{R}^{2}\right) \hookrightarrow$ $L^{\infty}\left(\mathbb{R}^{2}\right)$ :

$$
\begin{aligned}
& 2^{2 j s}\left\|\theta_{j}\right\|_{L_{T}^{\infty} L_{x}^{2}}^{2}+2^{2 j(s+1)}\left\|\theta_{j}\right\|_{L_{T}^{2} L_{x}^{2}}^{2} \leq 2^{2 j s}\left\|\left(\theta_{0}\right)_{j}\right\|_{L_{x}^{2}}^{2} \\
& \quad+C\left(\kappa_{*}, s\right)\left(l_{j}^{\prime}\right)^{2} \int_{0}^{T}\|u\|_{H_{x}^{s-1}}^{2}\|\nabla \theta\|_{H_{x}^{s-1}}^{2} \mathrm{~d} t \\
& \quad+C\left(\kappa_{*}, a,\|\theta\|_{L_{T}^{\infty} L_{x}^{\infty}}\right)\left(l_{j}^{\prime}\right)^{2} \int_{0}^{T}\|\nabla \theta\|_{L_{x}^{\infty}}^{2}\|\nabla \theta\|_{H_{x}^{s-1}}^{2} \mathrm{~d} t, \quad j \geq 0,
\end{aligned}
$$

which, together with the $L^{2}$-Estimate (2.45) and the Gronwall's inequality, implies (1.21).

For $s \geq 3$, we make use of the following commutator estimate

$$
\left\|\left[u, \Delta_{j}\right] \nabla \theta\right\|_{L_{x}^{2}} \leq C l_{j}^{\prime} 2^{j(1-s)}\left(\|\nabla u\|_{L_{x}^{\infty}}\|\nabla \theta\|_{H_{x}^{s-2}}+\|\nabla u\|_{H_{x}^{s-2}}\|\nabla \theta\|_{L_{x}^{\infty}}\right),
$$

such that the estimate (1.22) follows. 
Case $\left(\theta_{0}, u_{0}\right) \in H^{s-1}\left(\mathbb{R}^{2}\right) \times\left(H^{s}\left(\mathbb{R}^{2}\right)\right)^{2}, s>2$

We recall the preliminary estimate for $u_{j}$ in (2.49). We apply Lemma 2.1 to derive the following commutator estimates for $s \in(2,3)$ and $\nu \in(s-2,1) \subset(0,1)$

$$
\begin{aligned}
& \left\|\left[u, \Delta_{j}\right] \nabla u\right\|_{L_{x}^{2}} \leq C l_{j} 2^{j(1-s)}\|\nabla u\|_{H_{x}^{\nu}}\|\nabla u\|_{H_{x}^{s-1-\nu}}, \\
& \quad 2^{j}\left\|\left[\mu, \Delta_{j}\right] \nabla u\right\|_{L_{x}^{2}} \leq C l_{j}^{\prime} 2^{j(1-s)}\left(\|\nabla \mu\|_{L_{x}^{\infty}}\|\nabla u\|_{H_{x}^{s-1}}+\|\nabla \mu\|_{H_{x}^{s-1}}\|\nabla u\|_{L_{x}^{\infty}}\right),
\end{aligned}
$$

which implies then

$$
\begin{aligned}
& 2^{2 j s}\left\|u_{j}\right\|_{L_{T}^{\infty} L_{x}^{2}}^{2}+2^{2 j(s+1)}\left\|u_{j}\right\|_{L_{T}^{2} L_{x}^{2}}^{2} \leq 2^{2 j s}\left\|\left(u_{0}\right)_{j}\right\|_{L_{x}^{2}}^{2}+C\left(\mu_{*}\right) \int_{0}^{T} 2^{2 j(s-1)}\left\|\theta_{j}\right\|_{L_{x}^{2}}^{2} \mathrm{~d} t \\
& \quad+C\left(\mu_{*}, s, \nu\right)\left(l_{j}\right)^{2} \int_{0}^{T}\|\nabla u\|_{H_{x}^{\nu}}^{2}\|\nabla u\|_{H_{x}^{s-1-\nu}}^{2} \mathrm{~d} t \\
& \quad+C\left(\mu_{*}, s,\|b\|_{C^{[s]+1}},\|\theta\|_{L_{T}^{\infty} H_{x}^{1}}\right)\left(l_{j}^{\prime}\right)^{2} \int_{0}^{T}\|\nabla \theta\|_{L_{x}^{\infty}}^{2}\|\nabla u\|_{H_{x}^{s-1}}^{2}+\|\nabla \theta\|_{H_{x}^{s-1}}^{2}\|\nabla u\|_{L_{x}^{\infty}}^{2} \mathrm{~d} t, \quad j \geq 0 .
\end{aligned}
$$

This, together with the $L^{2}$-Estimate (2.51) and Sobolev's embedding $H^{s-1}\left(\mathbb{R}^{2}\right) \hookrightarrow L^{\infty}\left(\mathbb{R}^{2}\right)$, implies $(1.23)$ where $\nu \in(0,1)$ is taken to be a small constant bigger than $s-2$.

For $s \geq 3$, we use the commutator estimate (2.52) with $\theta$ replaced by $u$, to arrive at (1.24).

\section{Acknowledgements}

The authors would like to thank the referee for his/her valuable comments.

Funding Open Access funding enabled and organized by Projekt DEAL.

Open Access. This article is licensed under a Creative Commons Attribution 4.0 International License, which permits use, sharing, adaptation, distribution and reproduction in any medium or format, as long as you give appropriate credit to the original author(s) and the source, provide a link to the Creative Commons licence, and indicate if changes were made. The images or other third party material in this article are included in the article's Creative Commons licence, unless indicated otherwise in a credit line to the material. If material is not included in the article's Creative Commons licence and your intended use is not permitted by statutory regulation or exceeds the permitted use, you will need to obtain permission directly from the copyright holder. To view a copy of this licence, visit http://creativecommons.org/licenses/by/4.0/.

Publisher's Note Springer Nature remains neutral with regard to jurisdictional claims in published maps and institutional affiliations.

\section{References}

[1] Abidi, H., Zhang, P.: On the global well-posedness of 2-D inhomogeneous incompressible Navier-Stokes system with variable viscous coefficient. J. Differ. Equ. 259, 3755-3802 (2015)

[2] Abidi, H., Zhang, P.: On the global well-posedness of 2-D Boussinesq system with variable viscosity. Adv. Math. 305, 1202-1249 (2017)

[3] Antontsev, S.N., Kazhikhov, A.V., Monakhov, V.N.: Boundary Value Problems in Mechanics of Nonhomogeneous Fluids. Studies in Mathematics and its Applications, vol. 22. North-Holland Publishing Co., Amsterdam (1990). Translated from the Russian

[4] Bahouri, H., Chemin, J.-Y., Danchin, R.: Fourier Analysis and Nonlinear Partial Differential Equations. Springer, Berlin (2010) 
[5] Boussinesq, J.: Théorie des ondes et des remous qui se propagent le long d'un canal rectangulaire horizontal en communiquant au liquide contenu dans ce canal des vitesses sensiblement pareilles de la surface au fond. J. Math. Pures Appl. (2) 17, 55-108 (1872)

[6] Brandolese, L., Schonbek, M.E.: Large time decay and growth for solutions of a viscous Boussinesq system. Trans. Am. Math. Soc. 364, 5057-5090 (2012)

[7] Cannon, J.R., DiBenedetto, E.: The Initial Value Problem for the Boussinesq Equations with Data in $L^{p}$. Lecture Notes in Mathematics, vol. 771, pp. 129-144. Springer, Berlin (1980)

[8] Cao, C., Wu, J.: Global regularity for the two-dimensional anisotropic Boussinesq equations with vertical dissipation. Arch. Ration. Mech. Anal. 208, 985-1004 (2013)

[9] Chae, D.: Global regularity for the 2D Boussinesq equations with partial viscosity terms. Adv. Math. 203, 497-513 (2006)

[10] Chae, D., Nam, H.S.: Local existence and blow-up criterion for the Boussinesq equations. Proc. R. Soc. Edinb. Sect. A 127, 935-946 (1997)

[11] Danchin, R.: Remarks on the lifespan of the solutions to some models of incompressible fluid mechanics. Proc. Am. Math. Soc. 141, 1979-1993 (2013)

[12] Danchin, R., Liao, X.: On the well-posedness of the full low Mach number limit system in general critical Besov spaces. Commun. Contemp. Math. 14, 1250022 (2012)

[13] Danchin, R., Paicu, M.: Global existence results for the anisotropic Boussinesq system in dimension two. Math. Models Methods Appl. Sci. 21, 421-457 (2011)

[14] Deng, W., Wu, J., Zhang, P.: Stability of Couette flow for 2D Boussinesq system with vertical dissipation. J. Funct. Anal. 281(12), Paper No. 109255 (2021)

[15] Diaz, J.I., Galiano, G.: Existence and uniqueness of solutions of the Boussinesq system with nonlinear thermal diffusion. Topol. Methods Nonlinear Anal. 11, 59-82 (1998)

[16] Desjardins, B.: Regularity results for two-dimensional flows of multiphase viscous fluids. Arch. Ration. Mech. Anal. 137, 135-158 (1997)

[17] Duvaut, G., Lions, J.L.: Transfert de chaleur dans un fluide de Bingham dont la viscosité dépend de la température. J. Funct. Anal. 11, 93-110 (1972)

[18] Elgindi, T.: Finite-time singularity formation for $C^{1, \alpha}$ solutions to the incompressible Euler equations on $\mathbb{R}^{3}(2019)$. arXiv:1904.04795

[19] Elgindi, T., Jeong, I.: Finite-time singularity formation for strong solutions to the Boussinesq system. Ann. PDE 6, 2524-5317 (2020)

[20] Feireisl, E., Málek, J.: On the Navier-Stokes equations with temperature-dependent transport coefficients. Differ. Equ. Nonlinear Mech. pp. 1-14 (2006)

[21] Gaedtke, M., Abishek, S., Mead-Hunter, R., King, A., Mullins, B., Nirschl, H., Krause, M.: Total enthalpy-based lattice Boltzmann simulations of melting in paraffin/metal foam composite phase change materials. Int. J. Heat Mass Transf. $155,119870(2020)$

[22] Gill, A.: Atmosphere-Ocean Dynamics. International Geophysics Series, vol. 30. Academic Press, New York (1982)

[23] Goncharova, O.N.: Uniqueness of the solution of a two-dimensional nonstationary problem for convection equations with temperature-dependent viscosity. Differ. Uravn. 38, 234-242 (2002). (286)

[24] Hmidi, T., Keraani, S.: On the global well-posedness of the Boussinesq system with zero viscosity. Indiana Univ. Math. J. 58, 1591-1618 (2009)

[25] Hmidi, T., Keraani, S., Rousset, F.: Global well-posedness for a Boussinesq-Navier-Stokes system with critical dissipation. J. Differ. Equ. 249, 2147-2174 (2010)

[26] Hmidi, T., Keraani, S., Rousset, F.: Global well-posedness for Euler-Boussinesq system with critical dissipation. Commun. Partial Differ. Equ. 36, 420-445 (2011)

[27] Hou, T.Y., Li, C.: Global well-posedness of the viscous Boussinesq equations. Discrete Contin. Dyn. Syst. 12, 1-12 (2005)

[28] Ladyženskaja, O.A., Solonnikov, V.A.: The unique solvability of an initial-boundary value problem for viscous incompressible inhomogeneous fluids. Zap. Naučn. Sem. Leningrad. Otdel. Mat. Inst. Steklov. (LOMI) 52, 52-109, 218-219 $(1975)$

[29] Liao, X.: A global existence result for a zero Mach number system. J. Math. Fluid Mech. 16, 77-103 (2014)

[30] Liao, X., Zillinger, C.: On variable viscosity and enhanced dissipation. arXiv:2110.10976

[31] Lions, P.L.: Mathematical Topics in Fluid Mechanics, Vol. 1. Oxford Lecture Series in Mathematics and its Applications, vol. 3, p. xiv +237. The Clarendon Press, New York (1996)

[32] Lide, D. (ed.): CRC Handbook of Chemistry and Physics, Internet Version 2005. CRC Press, Boca Raton (2005). http://www.hbcpnetbase.com

[33] Lorca, S., Boldrini, J.: The initial value problem for a generalized Boussinesq model. Nonlinear Anal. 36, 457-480 (1999)

[34] Majda, A.: Introduction to PDEs and Waves for the Atmosphere and Ocean. Courant Lecture Notes in Mathematics, vol. 9. AMS/CIAM (2003) 
[35] Majda, A., Bertozzi, A.: Vorticity and Incompressible Flow. Cambridge University Press, Cambridge (2002)

[36] Moffatt, H.K.: Some remarks on topological fluid mechanics. In: Ricca, R.L. (ed.) An Introduction to the Geometry and Topology of Fluid Flows, pp. 3-10. Kluwer Academic Publishers, Dordrecht (2001)

[37] Pérez, C., Thomas, J.-M., Blancher, S., Creff, R.: The steady Navier-Stokes/energy system with temperature-dependent viscosity. I. Analysis of the continuous problem \& II. The discrete problem and numerical experiments. Int. J. Numer. Methods Fluids 56, 63-89 \& 91-114 (2008)

[38] Rodrigues, J.F.: Weak solutions for thermoconvective flows of Boussinesque-Stefan type. In: Mathematical Topics in Fluid Mechanics (Lisbon, 1991). Pitman Research Notes in Mathematics Series, vol. 274, pp. 93-116 (1992)

[39] Sun, Y., Zhang, Z.: Global regularity for the initial-boundary value problem of the 2-D Boussinesq system with variable viscosity and thermal diffusivity. J. Differ. Equ. 255, 1069-1085 (2013)

[40] Tao, L., Wu, J., Zhao, K., Zheng, X.: Stability near hydrostatic equilibrium to the 2D Boussinesq equations without thermal diffusion. Arch. Ration. Mech. Anal. 237, 585-630 (2020)

[41] Wang, C., Zhang, Z.: Global well-posedness for the 2-D Boussinesq system with the temperature-dependent viscosity and thermal diffusivity. Adv. Math. 228, 43-62 (2011)

[42] Weinan, E., Shu, C.: Small-scale structures in Boussinesq convection. Phys. Fluids 6, 49-58 (1994)

[43] Wu, J.: The 2D Incompressible Boussinesq Equations, Peking University Summer School Lecture Notes

[44] Zillinger, C.: On enhanced dissipation for the Boussinesq equations. J. Differ. Equ. 282, 407-445 (2021)

Zihui He and Xian Liao

Institute of Analysis

Karlsruhe Institute of Technology

Englerstrasse 2

76131 Karlsruhe

Germany

e-mail: xian.liao@kit.edu

Zihui He

e-mail: zihui.he@kit.edu

(Received: July 10, 2021; revised: October 28, 2021; accepted: November 1, 2021) 